Tohoku Math. J.

57 (2005), 447-468

\title{
THE EVOLUTION OF PERIODIC POPULATION SYSTEMS UNDER RANDOM ENVIRONMENTS
}

\author{
Nguyen HuU Du, Ryusuke Kon, Kazunori Sato \\ AND YASUHIRO TAKEUCHI
}

(Received March 4, 2003, revised May 11, 2005)

\begin{abstract}
In this paper we study the behavior of trajectories of the Lotka-Volterra competition system with periodic coefficients under telegraph noise. We give sufficient conditions for the average permanence. Furthermore, we determine the $\omega$-limit sets of the system.
\end{abstract}

1. Introduction. In this paper we study the behavior of trajectories of the LotkaVolterra competition system with periodic coefficients under telegraph noise. Until now, many models have revealed the effect of environmental variability on the population dynamics in mathematical ecology [10, 14]. In particular, a great effort has been made to find the possibility of the coexistence of competing species under the unpredictable or rather predictable (such as seasonal) environmental fluctuations. It is well recognized that the seasonality has similar effects to stochastic variation [4, 9], but as Loreau [11] pointed out, the theory of coexistence in a seasonal environment needs further development to reveal the variety of possibilities that seasonal fluctuations may cause. Among these, Namba and Takahashi [13] review the results on Lotka-Volterra competition systems with periodic coefficients, and show the new modes of the possibilities of stable periodic solutions even when the stable coexistence cannot be realized in the corresponding classical Lotka-Volterra system with constant coefficients.

Here, we restrict the competition parameters so that there is no possibility of the multiple periodic solutions that [13] shows. Then we consider the situation where the interacting populations experience pseudo-stochastic environmental fluctuations with unpredictable discontinuous change, such as seasonality in a year with 'a cycle of three cold days and four warm days'. In a separate paper [6], we analyze the Lotka-Volterra competition system with constant coefficients under telegraph noises, i.e., environmental variability causes parameter switching between two systems.

Our focus of attention is on the intermediate case where environments have both predictable and unpredictable aspects. This case is studied by using a combined system of two Lotka-Volterra systems with periodic coefficients. In this system, it is assumed that at every moment the population dynamics is governed by one of the two Lotka-Volterra systems with periodic coefficients. That is, the populations usually experience predictable changes of environments. However, it is also assumed that the population dynamics abruptly becomes governed by another Lotka-Volterra system. This abrupt switch between two systems occurs

2000 Mathematics Subject Classification. Primary 34C12; Secondary 60H10, 92D25.

key words and phrases. Lotka-Volterra equation, competition, bistable, telegraph noise, average permanence. 
in an unpredictable manner. More precisely, the waiting time for the occurrence of the switch follows an exponential distribution. These assumptions, for example, correspond to the situation where environments change seasonally and there are both warm days and cold days in each season. The purpose of this paper is to show that under this situation our system exhibits complex behavior due to the mutual interference between stochastic and periodic dynamics.

The paper is organized as follows. Section 2 is devoted to some fundamental properties of the competition Lotka-Volterra equation with periodic coefficients under telegraph noise. In particular, we give sufficient conditions to ensure the average permanence of the equation, i.e., the coexistence of two species in the sense that the time averages of the both species are positive (Theorem 2.3). The two subsequent sections deal with the Lotka-Volterra competition system composed of two stable subsystems or of one stable and one bistable subsystem, respectively. In Section 3, it is proved that the $\omega$-limit sets of the system include periodic solutions of the subsystems (Theorem 3.6). Moreover, it is also shown that the $\omega$-limit sets include every orbit starting at a point on the periodic solutions of the subsystems (Theorem 3.8). In Section 4, it is proved that the $\omega$-limit sets of the system include a periodic solution of the stable subsystem (Theorem 4.7) and that the position of the periodic solution of the stable subsystem and the 'neutral curve' of the bistable subsystem is essential in determining the shape of the $\omega$-limit sets of the system (Theorem 4.9). Biological and mathematical implications are discussed in the final section.

2. Preliminary discussion. Let $(\Omega, \mathcal{F}, \boldsymbol{P})$ be a probability space satisfying the general hypotheses [12] and let $\left(\xi_{t}\right)_{t \geq 0}$ be a Markov process, defined on $(\Omega, \mathcal{F}, \boldsymbol{P})$ and taking values in the set of two elements, say $E=\{+,-\}$. Suppose that $\left(\xi_{t}\right)$ has the transition intensities $+\stackrel{\alpha}{\rightarrow}-$ and $-\stackrel{\beta}{\rightarrow}+$ with $\alpha>0, \beta>0$. The process $\left(\xi_{t}\right)$ has a unique stationary distribution

$$
p=\lim _{t \rightarrow \infty} \boldsymbol{P}\left\{\xi_{t}=+\right\}=\frac{\beta}{\alpha+\beta}, \quad q=\lim _{t \rightarrow \infty} \boldsymbol{P}\left\{\xi_{t}=-\right\}=\frac{\alpha}{\alpha+\beta} .
$$

The trajectory of $\left(\xi_{t}\right)$ is a piecewise-constant, cadlag function. Suppose that

$$
0=\tau_{0}<\tau_{1}<\tau_{2}<\cdots<\tau_{n}<\cdots
$$

are its jump times. Put

$$
\sigma_{1}=\tau_{1}-\tau_{0}, \quad \sigma_{2}=\tau_{2}-\tau_{1}, \quad \ldots, \quad \sigma_{n}=\tau_{n}-\tau_{n-1}, \quad \ldots .
$$

Then $\sigma_{1}=\tau_{1}$ is the first exile from the initial state, $\sigma_{2}$ is the time that the process $\left(\xi_{t}\right)$ spends in the state into which it moves from the first state and so forth. It is known that the random variables $\sigma_{k}(k=1, \ldots, n)$ are mutually independent when a sequence $\left(\xi_{\tau_{k}}\right)_{k=1}^{n}$ is given (see [8, vol. 2, p. 217]). Note that if $\xi_{0}$ is given, then $\xi_{\tau_{n}}$ is constant, since the process $\left(\xi_{t}\right)$ takes only two values. Hence, $\left(\sigma_{k}\right)_{n=1}^{\infty}$ is a sequence of conditionally independent random variables, valued in $[0, \infty]$. Moreover, if $\xi_{0}=+$, then $\sigma_{2 n+1}$ has the exponential density $\alpha 1_{[0, \infty)} \exp (-\alpha t)$ and $\sigma_{2 n}$ has the density $\beta 1_{[0, \infty)} \exp (-\beta t)$. Conversely, if $\xi_{0}=-$, then $\sigma_{2 n}$ has the exponential density $\alpha 1_{[0, \infty)} \exp (-\alpha t)$, and $\sigma_{2 n+1}$ has the density $\beta 1_{[0, \infty)} \exp (-\beta t)$ (see [8, vol. 2, p. 217]). Here $1_{[0, \infty)}=1$ for $t \geq 0\left(1_{[0, \infty)}=0\right.$ for $\left.t<0\right)$. 
Let $\mathcal{F}_{0}^{n}=\sigma\left(\tau_{k}: k \leq n\right)$ and $\mathcal{F}_{n}^{\infty}=\sigma\left(\tau_{k}-\tau_{n}: k>n\right)$. We see that $\mathcal{F}_{0}^{n}$ is independent of $\mathcal{F}_{n}^{\infty}$ for any $n \in N$ under the condition that $\xi_{0}$ is given.

Let $\xi_{0}$ have the distribution $\boldsymbol{P}\left\{\xi_{0}=+\right\}=p$ and $\boldsymbol{P}\left\{\xi_{0}=-\right\}=q$. Then $\left(\xi_{t}\right)$ is a stationary process. Therefore, there exists a group $\theta^{t}, t \in \boldsymbol{R}$, of $\boldsymbol{P}$-measure preserving transformations $\theta^{t}: \Omega \rightarrow \Omega$ such that $\xi_{t}(\omega)=\xi_{0}\left(\theta^{t} \omega\right), \omega \in \Omega$.

We consider the competition Lotka-Volterra equation

$$
\left\{\begin{array}{l}
\dot{x}=x\left(a\left(\xi_{t}, t\right)-b\left(\xi_{t}, t\right) x-c\left(\xi_{t}, t\right) y\right) \\
\dot{y}=y\left(d\left(\xi_{t}, t\right)-e\left(\xi_{t}, t\right) x-f\left(\xi_{t}, t\right) y\right)
\end{array}\right.
$$

where $g: E \times \boldsymbol{R} \rightarrow \boldsymbol{R}_{+}$for $g=a, b, c, d, e, f$ such that $g(i, \cdot)$ are continuous and periodic functions with period $T>0$ for any $i \in E$. Suppose that $m$ and $M$ are two positive constants such that

$$
m \leq g(i, t) \leq M, \quad i \in E, t \in \boldsymbol{R}, \quad \text { for } g=a, b, c, d, e, f .
$$

The process $\left(\xi_{t}\right)$ interferes in (2.4) as a noise which is well-known as a real noise form (or multiplicative noise, see [1]). Without the noise $\left(\xi_{t}\right)$, i.e., $g\left(\xi_{t}, t\right)=g(t)$ for any $g=$ $a, b, c, d, e, f,(2.4)$ is well studied (see [7] for example). In that case, it is proved that under certain conditions posed on the coefficients, either (2.4) has a unique periodic solution which attracts all solutions starting in $(0, \infty) \times(0, \infty)$ or every solution of $(2.4)$, except the solution starting at the neutral curve, has a component tending to 0 (see $[2,3,6,7]$ ).

In the case when the noise $\left(\xi_{t}\right)$ intervenes virtually into (2.4), it makes a switching between the deterministic periodic system

$$
\left\{\begin{array}{l}
\dot{x}^{+}(t)=x^{+}(t)\left(a(+, t)-b(+, t) x^{+}(t)-c(+, t) y^{+}(t)\right) \\
\dot{y}^{+}(t)=y^{+}(t)\left(d(+, t)-e(+, t) x^{+}(t)-f(+, t) y^{+}(t)\right)
\end{array}\right.
$$

and the deterministic periodic one

$$
\left\{\begin{array}{l}
\dot{x}^{-}(t)=x^{-}(t)\left(a(-, t)-b(-, t) x^{-}(t)-c(-, t) y^{-}(t)\right) \\
\dot{y}^{-}(t)=y^{-}(t)\left(d(-, t)-e(-, t) x^{-}(t)-f(-, t) y^{-}(t)\right) .
\end{array}\right.
$$

Thus, the relationship between these two systems will determine the behavior of trajectories of (2.4).

As is known, the property of solutions of Lotka-Volterra systems near the boundary is dependent on two marginal equations. So, first we study the equations

$$
\begin{aligned}
\dot{u} & =u\left(a\left(\xi_{t}, t\right)-b\left(\xi_{t}, t\right) u\right), & u(0) \in \boldsymbol{R}^{+}, \\
\dot{v} & =v\left(d\left(\xi_{t}, t\right)-f\left(\xi_{t}, t\right) v\right), & v(0) \in \boldsymbol{R}^{+} .
\end{aligned}
$$

If $u(t)$ is a solution of (2.7) and $v(t)$ is a solution of (2.8), then $\left(\xi_{t}, u(t)\right)$ and $\left(\xi_{t}, v(t)\right)$ are Markov processes.

A random process $\left(\phi_{t}\right)$, valued in a measurable space $(S, \mathcal{S})$, is said to be periodic with period $T$ if for any $t_{1}, t_{2}, \ldots, t_{n} \in \boldsymbol{R}$, the simultaneous distribution of $\left(\phi_{t_{1}+k T}, \phi_{t_{2}+k T}, \ldots\right.$, $\left.\phi_{t_{n}+k T}\right)$ does not depend on $k \in N$. We show that (2.7) has a unique solution $u^{*}(t)$ such that 
$\left(\xi_{t}, u^{*}(t)\right)$ is a periodic process. Indeed, put

$$
u^{*}(t)=\frac{\exp \{A(t)\}}{\int_{-\infty}^{t} b\left(\xi_{s}, s\right) \exp \{A(s)\} d s},
$$

where $A(t)=\int_{0}^{t} a\left(\xi_{s}, s\right) d s$. First, we see that

$$
\begin{aligned}
u^{*}(t+T, \omega) & =\frac{\exp \left\{\int_{0}^{t+T} a\left(\xi_{s}(\omega), s\right) d s\right\}}{\int_{-\infty}^{t+T} b\left(\xi_{s}(\omega), s\right) \exp \left\{\int_{0}^{s} a\left(\xi_{\tau}(\omega), \tau\right) d \tau\right\} d s} \\
& =\frac{\exp \left\{\int_{0}^{t+T} a\left(\xi_{s-T}\left(\theta^{T} \omega\right), s-T\right) d s\right\}}{\int_{-\infty}^{t+T} b\left(\xi_{s-T}\left(\theta^{T} \omega\right), s-T\right) \exp \left\{\int_{0}^{s} a\left(\xi_{\tau-T}\left(\theta^{T} \omega\right), \tau-T\right) d \tau\right\} d s} \\
& =\frac{\exp \left\{\int_{-T}^{t} a\left(\xi_{s}\left(\theta^{T} \omega\right), s\right) d s\right\}}{\exp \left\{\int_{-T}^{0} a\left(\xi_{s}\left(\theta^{T} \omega, s\right) d s\right\} \int_{-\infty}^{t} b\left(\xi_{s}\left(\theta^{T} \omega\right), s\right) \exp \left\{\int_{0}^{s} a\left(\xi_{\tau}\left(\theta^{T} \omega\right), \tau\right) d \tau\right\} d s\right.} \\
& =\frac{\exp \left\{\int_{0}^{t} a\left(\xi_{s}\left(\theta^{T} \omega\right), s\right) d s\right\}}{\int_{-\infty}^{t} \exp \left\{\int_{0}^{s} a\left(\xi_{\tau}\left(\theta^{T} \omega\right), \tau\right) d \tau\right\} b\left(\xi_{s}\left(\theta^{T} \omega\right), s\right) d s}=u^{*}\left(t, \theta^{T} \omega\right) .
\end{aligned}
$$

Hence, by virtue of the $\boldsymbol{P}$-measure preserving property of $\theta$, for any continuous function $h$, for any $t_{1}<t_{2}<\cdots<t_{n}$ and for each $k \in N$ we have

$$
\begin{aligned}
& \boldsymbol{E}\left[h\left(\xi_{t_{1}+k T}, u^{*}\left(t_{1}+k T\right), \xi_{t_{2}+k T}, u^{*}\left(t_{2}+k T\right), \ldots, \xi_{t_{n}+k T}, u^{*}\left(t_{n}+k T\right)\right)\right] \\
& \quad=\boldsymbol{E}\left[h\left(\xi_{t_{1}}\left(\theta^{k T}\right), u^{*}\left(t_{1}, \theta^{k T}\right), \xi_{t_{2}}\left(\theta^{k T}\right), u^{*}\left(t_{2}, \theta^{k T}\right), \ldots, \xi_{t_{n}}\left(\theta^{k T}\right), u^{*}\left(t_{n}, \theta^{k T}\right)\right)\right] \\
& \quad=\boldsymbol{E}\left[h\left(\xi_{t_{1}}(\cdot), u^{*}\left(t_{1}, \cdot\right), \xi_{t_{2}}(\cdot), u^{*}\left(t_{2}, \cdot\right), \ldots, \xi_{t_{n}}(\cdot), u^{*}\left(t_{n}, \cdot\right)\right)\right]
\end{aligned}
$$

This means that $\left(\xi_{t}, u^{*}(t)\right)$ is a periodic process with period $T$. Similarly, (2.8) has a unique solution $v^{*}(t)$ given by

$$
v^{*}(t)=\frac{\exp \{D(t)\}}{\int_{-\infty}^{t} f\left(\xi_{s}, s\right) \exp \{D(s)\} d s}, \quad D(t)=\int_{0}^{t} d\left(\xi_{s}, s\right) d s
$$

such that $\left(\xi_{t}, v^{*}(t)\right)$ is a Markov periodic process. The uniqueness follows from the next lemma.

LEMMA 2.1. For any $u_{0}>0$ (resp. $\left.v_{0}>0\right), \lim _{t \rightarrow \infty}\left(u(t)-u^{*}(t)\right)=0$ a.s. (resp. $\lim _{t \rightarrow \infty}\left(v(t)-v^{*}(t)\right)=0$ a.s. $)$, where $u(t)$ is the solution of $(2.7)$ satisfying $u(0)=u_{0}$ (resp. $v(t)$ is the solution of (2.8) satisfying $\left.v(0)=v_{0}\right)$.

Proof. Put $z=1 / u-1 / u^{*}$. Then we have $\dot{z}=-a z$. Thus, since $z$ is bounded below by a positive constant, we obtain the result.

LEMMA 2.2 (Law of large numbers for periodic processes). For any continuous bounded function $h(t, i, u)$, periodic in $t$ with period $T$, we have

$$
\lim _{t \rightarrow \infty} \frac{1}{t} \int_{0}^{t} h\left(s, \xi_{s}, u^{*}(s)\right) d s=\boldsymbol{E}\left[\frac{1}{T} \int_{0}^{T} h\left(s, \xi_{s}, u^{*}(s)\right) d s\right] .
$$


Similarly,

$$
\lim _{t \rightarrow \infty} \frac{1}{t} \int_{0}^{t} h\left(s, \xi_{s}, v^{*}(s)\right) d s=\boldsymbol{E}\left[\frac{1}{T} \int_{0}^{T} h\left(s, \xi_{s}, v^{*}(s)\right) d s\right] .
$$

PROOF. Put

$$
X_{n}=\int_{n T}^{(n+1) T} h\left(s, \xi_{s}, u^{*}(s)\right) d s .
$$

Since $\left(\xi_{t}, u^{*}(t)\right)$ is periodic, $\left(X_{n}\right)$ is a stationary process. By the law of large numbers we have

$$
\lim _{n \rightarrow \infty} \frac{1}{n} \sum_{k=0}^{n} X_{k}=\boldsymbol{E}\left[X_{0} / \mathcal{J}\right] \text { a.s. , }
$$

where $\mathcal{J}$ is the $\sigma$-algebra of the invariant sets. However, $\left(\xi_{t}\right)$ is ergodic and $u^{*}(t)$ has no non-trivial invariant set, and hence we see that $\mathcal{J}=\{\emptyset, \Omega\}$. This implies that

$$
\begin{aligned}
\lim _{t \rightarrow \infty} \frac{1}{t} \int_{0}^{t} h\left(s, \xi_{s}, u^{*}(s)\right) d s & =\lim _{[t] \rightarrow \infty} \frac{1}{T} \frac{1}{[t / T]} \sum_{k=0}^{[t / T]} X_{k}=\frac{1}{T} \boldsymbol{E}\left[X_{0}\right] \\
& =\boldsymbol{E}\left[\frac{1}{T} \int_{0}^{T} h\left(s, \xi_{s}, u^{*}(s)\right) d s\right] .
\end{aligned}
$$

Here, $[x]$ denotes the integer such that $[x] \leq x<[x]+1$.

We study conditions that ensure the average permanence of (2.4).

THEOREM 2.3.

(a) If

$$
\lambda:=\frac{1}{T} \boldsymbol{E}\left[\int_{0}^{T}\left(a\left(\xi_{t}, t\right)-c\left(\xi_{t}, t\right) v^{*}(t)\right) d t\right]>0,
$$

then for any $x>0, y>0, \liminf _{t \rightarrow \infty}(1 / t) \int_{0}^{t} x(s, x, y) d s>0$ for $\boldsymbol{P}$-a.s.

(b) If

$$
\gamma:=\frac{1}{T} \boldsymbol{E}\left[\int_{0}^{T}\left(d\left(\xi_{t}, t\right)-e\left(\xi_{t}, t\right) u^{*}(t)\right) d t\right]>0,
$$

then for any $x>0, y>0, \liminf _{t \rightarrow \infty}(1 / t) \int_{0}^{t} y(s, x, y) d s>0$ for $\boldsymbol{P}$-a.s. Here $(x(t, x, y)$, $y(t, x, y))$ is a solution of (2.4).

Proof. (a) From the inequality $\dot{y}=y(d-e x-f y) \leq y(d-f y)$ it follows that if $y(0)=v(0)$, then $y(t) \leq v(t)$ for any $t>0$ by the comparison principle. On the other hand, by Lemma 2.1 we have $\lim _{t \rightarrow \infty}\left(v(t)-v^{*}(t)\right)=0$. Therefore, for any $\varepsilon>0$, there exists $t_{0}=t_{0}(\omega, \varepsilon)$ such that $v^{*}(t)+\varepsilon / M>v(t)$ for any $t>t_{0}$. Hence,

$$
\begin{aligned}
\frac{\dot{x}(t)}{x(t)} & =a\left(\xi_{t}, t\right)-b\left(\xi_{t}, t\right) x(t)-c\left(\xi_{t}, t\right) y(t) \\
& \geq a\left(\xi_{t}, t\right)-b\left(\xi_{t}, t\right) x(t)-c\left(\xi_{t}, t\right) v^{*}(t)-\varepsilon, \quad t>t_{0},
\end{aligned}
$$


which implies that

$$
\frac{1}{t} \int_{t_{0}}^{t} b\left(\xi_{s}, s\right) x(s) d s+\frac{\ln x(t)-\ln x\left(t_{0}\right)}{t} \geq \frac{1}{t} \int_{t_{0}}^{t}\left(a\left(\xi_{s}, s\right)-c\left(\xi_{s}, s\right) v^{*}(s)\right) d s-\varepsilon \frac{t-t_{0}}{t} .
$$

Since $x(t)$ is bounded, lim $\sup _{t \rightarrow \infty}\left(\ln x(t)-\ln x\left(t_{0}\right)\right) / t \leq 0$. By letting $t \rightarrow \infty$, we obtain

$$
\begin{aligned}
M \liminf _{t \rightarrow \infty} \frac{1}{t} \int_{0}^{t} x(s) d s & \geq \liminf _{t \rightarrow \infty}\left(\frac{1}{t} \int_{0}^{t} b\left(\xi_{s}, s\right) x(s) d s+\frac{\ln x(t)-\ln x\left(t_{0}\right)}{t}\right) \\
& \geq \liminf _{t \rightarrow \infty} \frac{1}{t} \int_{t_{0}}^{t}\left(a\left(\xi_{s}, s\right)-c\left(\xi_{s}, s\right) v^{*}(s)\right) d s-\varepsilon .
\end{aligned}
$$

Applying the law of large numbers (Lemma 2.2), we have

$$
\lim _{t \rightarrow \infty} \frac{1}{t} \int_{t_{0}}^{t}\left(a\left(\xi_{s}, s\right)-c\left(\xi_{s}, s\right) v^{*}(s)\right) d s=\frac{1}{T} \boldsymbol{E}\left[\int_{0}^{T}\left(a\left(\xi_{s}, s\right)-c\left(\xi_{s}, s\right) v^{*}(s)\right) d s\right] .
$$

Since $\varepsilon$ is arbitrary, it follows that

$$
\left.\liminf _{t \rightarrow \infty} \frac{1}{t} \int_{0}^{t} x(s) d s \geq \frac{1}{T M} \boldsymbol{E}\left[\int_{0}^{T} a\left(\xi_{s}, s\right)-c\left(\xi_{s}, s\right) v^{*}(s)\right) d s\right]=\frac{\lambda}{M}>0 .
$$

(b) Similarly,

$$
\liminf _{t \rightarrow \infty} \frac{1}{t} \int_{0}^{t} y(s) d s \geq \frac{\gamma}{M}>0
$$

REMARK 2.4.

(a) We note that the average permanence implies that $\limsup _{t \rightarrow \infty} x(t)>0$ and $\limsup _{t \rightarrow \infty} y(t)>0$.

(b) Conditions (2.10) and (2.11) are easily checked in a similar fashion based on the law of large numbers.

To get the further properties of the trajectories of the solutions of (2.4), we need additional hypotheses on the coefficients of (2.5) and (2.6). In Section 3, we assume both systems are stable. In Section 4, we assume that one of them is stable and the other is bistable.

\section{Dynamics of the stable subsystems.}

HYPOTHESIS 3.1. The coefficients of (2.5) and (2.6) satisfy

$$
\begin{gathered}
\sup _{0<t<T} \frac{a(i, t)}{b(i, t)}<\inf _{0<t<T} \frac{d(i, t)}{e(i, t)}, \\
\inf _{0<t<T} \frac{a(i, t)}{c(i, t)}>\sup _{0<t<T} \frac{d(i, t)}{f(i, t)}, \\
\inf _{0<t<T} \frac{b(i, t)}{e(i, t)}>\sup _{0<t<T} \frac{c(i, t)}{f(i, t)}
\end{gathered}
$$

for any $i \in E$. 
By (3.1) and (3.2), there are constants $k_{1}^{ \pm}, k_{2}^{ \pm}$such that

$$
\begin{array}{ll}
\frac{a(+, t)}{b(+, t)}<k_{1}^{+}<\frac{d(+, s)}{e(+, s)}, \quad \frac{a(+, t)}{c(+, t)}>k_{2}^{+}>\frac{d(+, s)}{f(+, s)}, \\
\frac{a(-, t)}{b(-, t)}<k_{1}^{-}<\frac{d(-, s)}{e(-, s)}, & \frac{a(-, t)}{c(-, t)}>k_{2}^{-}>\frac{d(-, s)}{f(-, s)}
\end{array}
$$

for any $t, s \in \boldsymbol{R}$ and $i \in E$. If $k_{1}^{+}=k_{1}^{-}$and $k_{2}^{+}=k_{2}^{-}$, by virtue of the results in $[2,3,5,7]$ with a slight modification, we can prove that there exists a unique periodic solution of (2.4) taking values in $(0, \infty) \times(0, \infty)$. Thus, we consider here only the case where either $k_{1}^{+} \neq$ $k_{1}^{-}$or $k_{2}^{+} \neq k_{2}^{-}$. Under these hypotheses, (2.5) (resp. (2.6)) has a unique periodic solution with period $T$ whose orbit, say $\gamma^{+}$(resp. $\gamma^{-}$), attracts any solution starting at a point in $(0, \infty) \times(0, \infty)$, that is,

$$
\lim _{t \rightarrow \infty} \rho\left(\left(x^{+}(t), y^{+}(t)\right), \gamma^{+}\right)=0 \quad\left(\text { resp. } \lim _{t \rightarrow \infty} \rho\left(\left(x^{-}(t), y^{-}(t)\right), \gamma^{-}\right)=0\right),
$$

where we define $\rho(x, A)=\inf \{\|x-z\| ; z \in A\}$.

From inequalities (3.4) and (3.5), we can choose $\varepsilon>0, \delta>0$ such that

$$
\begin{gathered}
a(+, t)-b(+, t) \varepsilon-c(+, t) k_{2}^{+}>\delta, \quad d(+, t)-e(+, t) k_{1}^{+}-f(+, t) \varepsilon>\delta, \\
a(-, t)-b(-, t) \varepsilon-c(-, t) k_{2}^{-}>\delta, \quad d(-, t)-e(-, t) k_{1}^{-}-f(-, t) \varepsilon>\delta
\end{gathered}
$$

for any $t>0$.

Let $r_{1}=\max \left\{k_{1}^{+}, k_{1}^{-}\right\}$and $r_{2}=\max \left\{k_{2}^{+}, k_{2}^{-}\right\}$. It is easy to see that the rectangle $\mathcal{K}_{0}=\left(0, r_{1}\right) \times\left(0, r_{2}\right)$ is a forward invariant set of $(2.4)$, which attracts all solutions $(x(t, x, y), y(t, x, y))$ of (2.4) with $x>0, y>0$. Therefore, we can suppose that $(x(t, x, y), y(t, x, y)) \in \mathcal{K}_{0}$ for any $t \geq 0$. Furthermore, by (3.7) and (3.8), we obtain that $\gamma^{+} \subset \mathcal{K}_{\varepsilon}$ and $\gamma^{-} \subset \mathcal{K}_{\varepsilon}$, where

$$
\mathcal{K}_{\zeta}=\left(\zeta, r_{1}\right) \times\left(\zeta, r_{2}\right)
$$

LEMMA 3.2. For any small $\delta_{1}>0, \delta_{2}>0$, there exists a $T_{1}^{*}=T_{1}^{*}\left(\delta_{1}, \delta_{2}\right)>0$ such that $\left(x^{i}(t), y^{i}(t)\right) \in U_{\delta_{1}}\left(\gamma^{i}\right)$ for any $t \geq T_{1}^{*}$, provided that $\left(x^{i}(0), y^{i}(0)\right) \in \mathcal{K}_{\delta_{2}}$. Here, $U_{\delta_{1}}\left(\gamma^{i}\right)$ is the $\delta_{1}$-neighborhood of $\gamma^{i}$ and $i \in E$.

PROOF. It follows from (3.6) that for any $(x, y) \in \overline{\mathcal{K}_{\delta_{2}}}$, there is $T(x, y)>0$ such that $\left(x^{+}(t, x, y), x^{+}(t, x, y)\right) \in U_{\delta_{1}}\left(\gamma^{+}\right)$and $\left(x^{-}(t, x, y), x^{-}(t, x, y)\right) \in U_{\delta_{1}}\left(\gamma^{-}\right)$for any $t>T(x, y)$. By the continuous dependence of solutions on the initial data, there is a neighborhood of $(x, y)$, denoted by $U_{x, y}$, such that $\left(x^{+}\left(t, x_{0}, y_{0}\right), y^{+}\left(t, x_{0}, y_{0}\right)\right) \in U_{\delta_{1}}\left(\gamma^{+}\right)$ and $\left(x^{-}\left(t, x_{0}, y_{0}\right), y^{-}\left(t, x_{0}, y_{0}\right)\right) \in U_{\delta_{1}}\left(\gamma^{-}\right)$for any $t>T(x, y)$ and $\left(x_{0}, y_{0}\right) \in U_{x, y}$. The family $\left\{U_{x, y} ;(x, y) \in \overline{\mathcal{K}_{\delta_{2}}}\right\}$ is an open covering of $\overline{\mathcal{K}_{\delta_{2}}}$. Since $\overline{\mathcal{K}_{\delta_{2}}}$ is compact, there is a finite family $\left\{U_{x_{1}, y_{1}}, U_{x_{2}, y_{2}}, \ldots, U_{x_{n}, y_{n}}\right\}$ such that $\mathcal{K}_{\delta_{2}} \subset \bigcup_{i=1}^{n} U_{x_{i}, y_{i}}$. By choosing $T_{1}^{*}=\max _{1 \leq i \leq n} T\left(x_{i}, y_{i}\right)$, we finish the proof of Lemma 3.2.

LEMMA 3.3. There is a $T_{2}^{*}>0$ such that $x^{i}\left(t^{*}\right) \leq k_{1}^{i}$ and $y^{i}\left(t^{*}\right) \leq k_{2}^{i}$ for a $t^{*} \in$ $\left[0, T_{2}^{*}\right]$, provided $\left(x^{i}(0), y^{i}(0)\right) \in \mathcal{K}_{0}$. Here $i \in E$. 
Proof. Since it is assumed that $\left(x^{i}(t, x, y), y^{i}(t, x, y)\right) \in \mathcal{K}_{0}$ for any $t \geq 0$, we consider the case $x^{i}(0) \leq r_{1}$ and $y^{i}(0)<r_{2}$. For $i \in E$, if $x^{i}(0) \leq k_{1}^{i}$, we take $t^{*}=0$. Suppose that $x^{i}(0)>k_{1}^{i}$. Whenever $x^{i}(t)>k_{1}^{i}$, we have $\dot{x}^{i}=x^{i}\left(a(i, t)-b(i, t) x^{i}-c(i, t) y^{i}\right) \leq$ $x^{i}\left(a(i, t)-k_{1}^{i} b(i, t)\right)$, which implies that

$$
x^{i}(t) \leq x^{i}(0) \exp \left\{\int_{0}^{t}\left(a(i, s)-k_{1}^{i} b(i, s)\right) d s\right\} \leq r_{1} \exp \left\{\int_{0}^{t}\left(a(i, s)-k_{1}^{i} b(i, s)\right) d s\right\} .
$$

Similarly, $y^{i}(t) \leq r_{2} \exp \left\{\int_{0}^{t}\left(d(i, s)-k_{2}^{i} f(i, s)\right) d s\right\}$. From (3.4) and (3.5) it follows that $\int_{0}^{\infty}\left(a(i, s)-k_{1}^{i} b(i, s)\right) d s=-\infty$ and $\int_{0}^{\infty}\left(d(i, s)-k_{2}^{i} f(i, s)\right) d s=-\infty$. Hence, we can choose

$$
\begin{aligned}
T_{2}^{*}=\inf \left\{t ; \exp \left\{\int_{0}^{t}\left(a(i, s)-k_{1}^{i} b(i, s)\right) d s\right\}<k_{1}^{i} / r_{1},\right. \\
\left.\exp \left\{\int_{0}^{t}\left(d(i, s)-k_{2}^{i} f(i, s)\right) d s\right\}<k_{2}^{i} / r_{2}, i \in E\right\}
\end{aligned}
$$

to get the result.

COROLlaRY 3.4. For any $0<\varepsilon_{1}<\varepsilon$ and $0 \leq t_{1}<t_{2}$, there exists an $\varepsilon_{2}\left(\varepsilon_{1}>\varepsilon_{2}>\right.$ $0)$ such that for any $i \in E$, the following hold.

(a) If $r_{2} \geq y^{i}\left(t_{1}\right) \geq \varepsilon_{1}$ (resp. $\left.r_{1} \geq x^{i}\left(t_{1}\right) \geq \varepsilon_{1}\right)$, then $r_{2} \geq y^{i}(t) \geq \varepsilon_{2}$ (resp. $r_{1} \geq$ $\left.x^{i}(t) \geq \varepsilon_{2}\right)$ for any $t>t_{1}$.

(b) If $\left(x^{i}\left(t_{1}\right), y^{i}\left(t_{1}\right)\right) \in\left[\varepsilon, r_{1}\right) \times\left[0, \varepsilon_{2}\right]$, then $x^{i}(t) \in\left[\varepsilon, r_{1}\right]$ for any $t>t_{1}$. Under the additional condition $y^{i}\left(t_{2}\right)<\varepsilon_{1}$ for some $t_{2}>t_{1}$, we have $\sup _{t_{1}<t<t_{2}} y^{i}(t) \leq \varepsilon_{1}$. A similar result holds for the case when $\left(x^{i}\left(t_{1}\right), y^{i}\left(t_{1}\right)\right) \in\left[0, \varepsilon_{2}\right] \times\left[\varepsilon, r_{2}\right)$.

PROOF. We prove for the case $i=+$ (for $i=-$, the proof is similar). We note that $y^{+}(t) \uparrow$ when $x^{+}(t) \leq k_{1}^{+}$and $y^{+}(t)<\varepsilon$. Further, if $x^{+}\left(t^{*}\right) \leq k_{1}^{+}$and $y^{+}\left(t^{*}\right)>\varepsilon$ for a $t^{*} \geq 0$, then $x^{+}(t) \leq k_{1}^{+}$and $y^{+}(t)>\varepsilon$ for any $t \geq t^{*}$. Therefore, by choosing $T_{2}^{*}$ as in Lemma 3.3, we have

$$
\inf _{t \geq t_{1}} y^{+}(t) \geq \min \left\{\inf _{t_{1} \leq t \leq t_{1}+T_{2}^{*}} y^{+}(t), \varepsilon\right\}
$$

It is obvious that

$$
\sup _{t_{2} \geq t \geq t_{1}} y^{+}(t)=\max \left\{\sup _{t_{1} \leq t \leq t_{1}+T_{2}^{*}} y^{+}(t), \sup _{t_{1}+T_{2}^{*} \leq t \leq t_{2}} y^{+}(t)\right\} .
$$

Set

$$
\varepsilon_{2}=\varepsilon_{1} \min \left\{\exp \left\{-M\left(r_{1}+r_{2}\right) T_{2}^{*}\right\}, \exp \left\{-M T_{2}^{*}\right\}\right\} .
$$

We have $\dot{y}^{+}=y^{+}\left(d(+, t)-e(+, t) x^{+}-f(+, t) y^{+}\right)>-y^{+} M\left(r_{1}+r_{2}\right)$. Then, if $y^{+}\left(t_{1}\right) \geq$ $\varepsilon_{1}$, we have

$$
\inf _{t_{1} \leq t \leq t_{1}+T_{2}^{*}} y^{+}(t)>y^{+}\left(t_{1}\right) \exp \left\{-M\left(r_{1}+r_{2}\right) T_{2}^{*}\right\}>\varepsilon_{1} \exp \left\{-M\left(r_{1}+r_{2}\right) T_{2}^{*}\right\} \geq \varepsilon_{2},
$$

which implies that $\inf _{t \geq t_{1}} y^{+}(t)>\varepsilon_{2}$, i.e., we obtain (a). 
Furthermore, if $\left(x^{+}\left(t_{1}\right), y^{+}\left(t_{1}\right)\right) \in\left[\varepsilon, r_{1}\right) \times\left[0, \varepsilon_{2}\right]$, then from inequalities (3.7) and (3.8) we obtain that $x^{+}(t)>\varepsilon$ for any $t>t_{1}$. Moreover, if $y^{+}\left(t_{2}\right) \leq \varepsilon_{1}$, then $\sup _{t_{1}+T_{2}^{*} \leq t \leq t_{2}} y^{+}(t) \leq \varepsilon_{1}$, and from $\dot{y}^{+}=y^{+}\left(d(+, t)-e(+, t) x^{+}-f(+, t) y^{+}\right)<y^{+} M$ we have $\sup _{t_{1}+T_{2}^{*} \geq t \geq t_{1}} y^{+}(t)<y^{+}\left(t_{1}\right) \exp \left\{M T_{2}^{*}\right\}<\varepsilon_{2} \exp \left\{M T_{2}^{*}\right\} \leq \varepsilon_{1}$, i.e., we obtain (b).

The proof of the other cases is similar and hence is omitted.

LEMMA 3.5. For any $i \in E$, let $\gamma_{\left[t_{1}, t_{2}\right]}^{i}$ denote the set $\left\{\left(x^{i}(t), y^{i}(t)\right) ; t_{1} \leq t \leq t_{2}\right\}$. Then, for any $\delta_{1}>0$, there is $\delta_{2}>0$ such that if $\left(x^{i}\left(t_{1}\right), y^{i}\left(t_{1}\right)\right) \in U_{\delta_{2}}\left(\gamma^{i}\right)$, we have $\gamma_{\left[t_{1}, t_{1}+2 T\right]}^{i} \cap U_{\delta_{1}}\left(x^{*}, y^{*}\right) \neq \emptyset$ for any $\left(x^{*}, y^{*}\right) \in \gamma^{i}$.

PROOF. This lemma follows from the continuous dependence of solutions on the initial data and the fact that $\gamma^{+}$(resp. $\gamma^{-}$) is the orbit of a periodic solution with period $T$.

THEOREM 3.6. Suppose that conditions (2.10), (2.11) and (3.1)-(3.3) hold. Let $\omega(x, y)$ be the $\omega$-limit set of the solution $(x(t, x, y), y(t, x, y))$ of $(2.4)$ with $x>0, y>0$. Then, $\gamma^{+}$and $\gamma^{-}$are two subsets of $\omega(x, y)$. (See Figure 1.)

PROOF. For the sake of convenience, we suppose that $\xi_{0}=+$. Put $\varepsilon_{1}=\min \{\varepsilon, \lambda /(2 M)$, $\gamma /(2 M)\}$. From Corollary 3.4, suppose that $\varepsilon_{2}=\varepsilon_{2}\left(\varepsilon_{1}\right)$ for (2.6) and $\varepsilon_{3}=\varepsilon_{3}\left(\varepsilon_{2}\right)$ for (2.5) are chosen. Set $\varepsilon_{4}=\min \left\{\varepsilon_{2}, \varepsilon_{3}\right\}$. Put

$$
x_{n}=x\left(\tau_{n}, x, y\right) \quad \text { and } \quad y_{n}=y\left(\tau_{n}, x, y\right) .
$$

We know that $\left(x_{n}, y_{n}\right)$ is $\mathcal{F}_{0}^{n}$-measurable for any $n$, since $\left(x_{n}, y_{n}\right)$ is completely determined by the sequence $\tau_{1}, \tau_{2}, \ldots, \tau_{n}$. With the convention that $\inf \{\varnothing\}=\infty$ and the assumption $\xi_{0}=+$, we construct a sequence

$$
\begin{aligned}
\eta_{1} & =\inf \left\{2 k+1 ;\left(x_{2 k+1} \geq \varepsilon \wedge y_{2 k+1} \geq \varepsilon_{4}\right) \text { or }\left(x_{2 k+1} \geq \varepsilon_{4} \wedge y_{2 k+1} \geq \varepsilon\right)\right\}, \\
\eta_{2} & =\inf \left\{2 k+1>\eta_{1} ;\left(x_{2 k+1} \geq \varepsilon \wedge y_{2 k+1} \geq \varepsilon_{4}\right) \text { or }\left(x_{2 k+1} \geq \varepsilon_{4} \wedge y_{2 k+1} \geq \varepsilon\right)\right\}, \\
& \vdots \\
\eta_{n} & =\inf \left\{2 k+1>\eta_{n-1} ;\left(x_{2 k+1} \geq \varepsilon \wedge y_{2 k+1} \geq \varepsilon_{4}\right) \text { or }\left(x_{2 k+1} \geq \varepsilon_{4} \wedge y_{2 k+1} \geq \varepsilon\right)\right\} .
\end{aligned}
$$

The random variables $\eta_{1}<\eta_{2}<\cdots<\eta_{k}<\cdots$ form a sequence of $\mathcal{F}_{0}^{n}$-stopping times (see [12]). Moreover, $\left\{\eta_{k}=n\right\} \in \mathcal{F}_{0}^{n}$ for any $k, n$. Thus, the event $\left\{\eta_{k}=n\right\}$ is independent of $\mathcal{F}_{n}^{\infty}$.

We show that $\eta_{n}<\infty$ a.s. for any $n$. Indeed, suppose to the contrary that there is $m \in N$ such that the set $\Gamma=\left\{\omega ; \eta_{m}=\infty\right.$ and $\left.\eta_{m-1}<\infty\right\}$ has a positive probability. Since for any $k$, either $x_{2 k+1} \geq \varepsilon$ or $y_{2 k+1} \geq \varepsilon$, if $\omega \in \Gamma$, then either $\left(x_{2 k+1}(\omega) \geq \varepsilon \wedge y_{2 k+1}(\omega)<\varepsilon_{4}\right)$ or $\left(x_{2 k+1}(\omega)<\varepsilon_{4} \wedge y_{2 k+1}(\omega) \geq \varepsilon\right)$ for any $2 k+1>\eta_{m-1}(\omega)$. Let $2 k+1>\eta_{m-1}$. Suppose that $x_{2 k+1} \geq \varepsilon$ and $y_{2 k+1}<\varepsilon_{4}$. By virtue of Corollary 3.4, $x_{2 k+2} \geq \varepsilon$. If $y_{2 k+2}>\varepsilon_{2}$, then by Corollary $3.4, x_{2 k+3} \geq \varepsilon_{2}$ and $y_{2 k+3}>\varepsilon_{3} \geq \varepsilon_{4}$, which is impossible (since either $x_{2 k+3}<\varepsilon_{4}$ or $y_{2 k+3}<\varepsilon_{4}$ ). Therefore, $x_{2 k+2} \geq \varepsilon$ and $y_{2 k+2} \leq \varepsilon_{2}$. Using Corollary 3.4 again, we see that $x_{2 k+3} \geq \varepsilon$, which implies that $y_{2 k+3}<\varepsilon_{4}$. Hence, if $x_{2 k+1} \geq \varepsilon$ and $y_{2 k+1}<\varepsilon_{4}$, then $x_{n} \geq \varepsilon$ and $y_{n}<\varepsilon_{2}$ for any $n>2 k+1$. By Corollary 3.4 we get $\sup _{t>\eta_{N-1}} y(t) \leq \varepsilon_{1}$. This contradicts $\lim \sup _{t \rightarrow \infty} y(t) \geq 2 \varepsilon_{1}>\varepsilon_{1}$. 

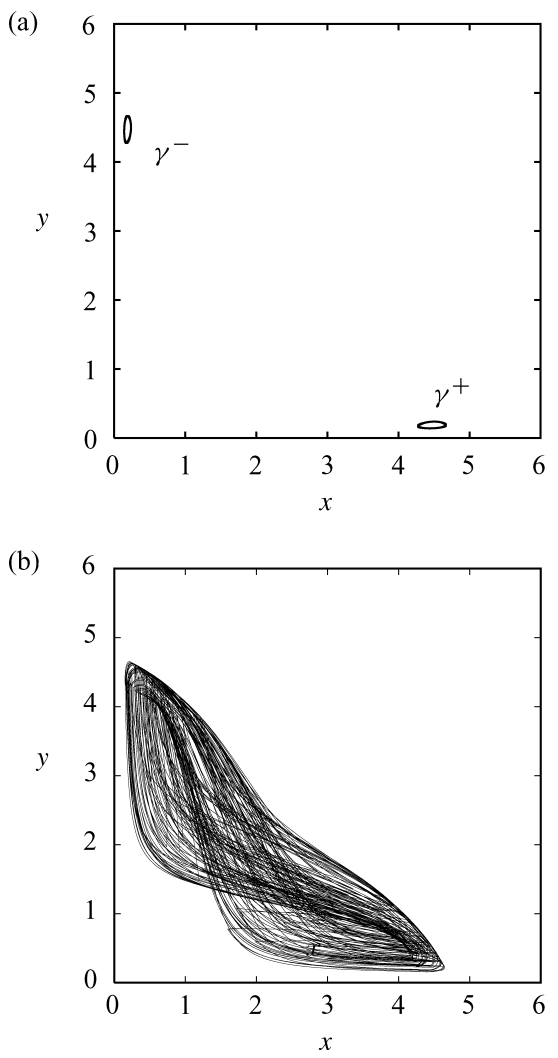

FIGURE 1. The $x-y$ phase planes. (a) The periodic solutions $\left(\gamma^{+}\right.$and $\left.\gamma^{-}\right)$of systems (2.5) and (2.6) with the following parameters are plotted: $a(+, t)=10, b(+, t)=0.1 \sin (t)+2.2, c(+, t)=1$, $d(+, t)=5, e(+, t)=1, f(+, t)=\sin (t+\pi / 2)+3, a(-, t)=5, b(-, t)=\sin (t+\pi / 2)+3$, $c(-, t)=1, d(-, t)=10, e(-, t)=1, f(-, t)=0.1 \sin (t)+2.2$. (b) The solution of system (2.4) switching between the above systems (2.5) and (2.6) with the initial condition $(x(0), y(0))=(4,0.2)$ is plotted for $t \in[700,1000]$. The transition intensities are $\alpha=1$ and $\beta=1$.

Let $U_{\delta_{1}}\left(\gamma^{-}\right)$be an arbitrary neighborhood of $\gamma^{-}$. We choose $\delta_{2}$ to be as in Lemma 3.5 and $T_{1}^{*}=T_{1}^{*}\left(\delta_{2}, \varepsilon_{4}\right)$ as in Lemma 3.2. Denote $A_{k}=\left\{\sigma_{\eta_{k}+1} \geq T_{1}^{*}+2 T\right\}$. We have

$$
\begin{aligned}
\boldsymbol{P}\left(\overline{A_{k}}\right) & =\boldsymbol{P}\left\{\sigma_{\eta_{k}+1}<T_{1}^{*}+2 T\right\} \\
& =\sum_{n=0}^{\infty} \boldsymbol{P}\left\{\sigma_{\eta_{k}+1}<T_{1}^{*}+2 T \mid \eta_{k}=2 n+1\right\} \boldsymbol{P}\left\{\eta_{k}=2 n+1\right\} \\
& =\sum_{n=0}^{\infty} \boldsymbol{P}\left\{\sigma_{2 n+2}<T_{1}^{*}+2 T \mid \eta_{k}=2 n+1\right\} \boldsymbol{P}\left\{\eta_{k}=2 n+1\right\}
\end{aligned}
$$




$$
\begin{aligned}
& =\sum_{n=0}^{\infty} \boldsymbol{P}\left\{\sigma_{2 n+2}<T_{1}^{*}+2 T\right\} \boldsymbol{P}\left\{\eta_{k}=2 n+1\right\} \\
& =\sum_{n=0}^{\infty} \boldsymbol{P}\left\{\sigma_{2}<T_{1}^{*}+2 T\right\} \boldsymbol{P}\left\{\eta_{k}=2 n+1\right\} \\
& =\boldsymbol{P}\left\{\sigma_{2}<T_{1}^{*}+2 T\right\}<1 .
\end{aligned}
$$

Similarly, we have

$$
\begin{aligned}
& \boldsymbol{P}\left(\overline{A_{k}} \cap\right.\left.\overline{A_{k+1}}\right) \\
&= \boldsymbol{P}\left\{\sigma_{\eta_{k}+1}<T_{1}^{*}+2 T, \sigma_{\eta_{k+1}+1}<T_{1}^{*}+2 T\right\} \\
&= \sum_{0 \leq l<n<\infty} \boldsymbol{P}\left\{\sigma_{\eta_{k}+1}<T_{1}^{*}+2 T, \sigma_{\eta_{k+1}+1}<T_{1}^{*}+2 T \mid \eta_{k}=2 l+1, \eta_{k+1}=2 n+1\right\} \\
& \quad \times \boldsymbol{P}\left\{\eta_{k}=2 l+1, \eta_{k+1}=2 n+1\right\}
\end{aligned}
$$

By induction we obtain

$$
\boldsymbol{P}\left(\bigcap_{i=k}^{n} \overline{A_{i}}\right)=\left(\boldsymbol{P}\left\{\sigma_{2}<T_{1}^{*}+2 T\right\}\right)^{n-k+1} \quad \text { for } n>k>0 .
$$

Hence,

$$
\boldsymbol{P}\left(\bigcap_{k=1}^{\infty} \bigcup_{i=k}^{\infty} A_{i}\right)=\boldsymbol{P}\left\{\omega ; \sigma_{\eta_{n}+1} \geq T_{1}^{*}+2 T \text { i.o. }\right\}=1,
$$

where 'i.o.' denotes 'infinitely often'. 
Therefore, there are infinitely many $n$ such that either $\left(x_{2 n+1} \geq \varepsilon \wedge y_{2 n+1} \geq \varepsilon_{4}\right)$ or $\left(x_{2 n+1} \geq \varepsilon_{4} \wedge y_{2 n+1} \geq \varepsilon\right)$ with $\sigma_{2 n+2} \geq T_{1}^{*}+2 T$. Hence, by using Lemma 3.2, we have $\left(x\left(\tau_{2 n+1}+T_{1}^{*}\right), y\left(\tau_{2 n+1}+T_{1}^{*}\right)\right)=\left(x^{-}\left(T_{1}^{*}, x_{2 n+1}, y_{2 n+1}\right), y^{-}\left(T_{1}^{*}, x_{2 n+1}, y_{2 n+1}\right)\right) \in$ $U_{\delta_{2}}\left(\gamma^{-}\right)$. Therefore, by Lemma 3.5, the solution $(x(t, x, y), x(t, x, y))$ enters the neighborhood $U_{\delta_{1}}\left(x^{*}, y^{*}\right)$ at a time in $\left(\tau_{2 n+1}+T_{1}^{*}, \tau_{2 n+1}+T_{1}^{*}+2 T\right)$. Thus, we conclude that $\gamma^{-} \subset \omega(x, y)$. The proof of the inclusion $\gamma^{+} \subset \omega(x, y)$ is quite similar.

Lemma 3.7. Let $\left(x^{*}, y^{*}\right) \in \gamma^{-}$and $\delta_{1}>0$. Then we have for $\left(x_{n}, y_{n}\right)$ defined by

$$
\boldsymbol{P}\left\{\left(x_{n}, y_{n}\right) \in U_{\delta_{1}}\left(x^{*}, y^{*}\right) \text { i.o. } n\right\}=1 .
$$

There is a similar result for the orbit $\gamma^{+}$.

Proof. Given $\xi_{0}=+$, let $\delta_{1}>0$ be arbitrary and let $\varepsilon_{1}, \varepsilon_{2}, \varepsilon_{3}, \varepsilon_{4}$ be as in the proof of Theorem 3.6. For any $(u, v) \in \mathcal{K}_{\varepsilon_{4}}$ we denote by $T(u, v)$ the first entrance time of the solution $\left(x^{-}(t, u, v), y^{-}(t, u, v)\right)$ into $U_{\delta_{1} / 2}\left(x^{*}, y^{*}\right)$, i.e.,

$$
T(u, v)=\inf \left\{t ;\left(x^{-}(t, u, v), y^{-}(t, u, v)\right) \in U_{\delta_{1} / 2}\left(x^{*}, y^{*}\right)\right\} .
$$

From Lemmas 3.2 and 3.5 we see that $T(u, v)<T_{1}^{*}\left(\delta_{2}, \varepsilon_{4}\right)+2 T$ with $\delta_{2}=\delta_{2}\left(\delta_{1} / 2\right)$ as mentioned in Lemma 3.5. Since the left-hand sides of (2.6) are bounded, there is $t_{1}>0$ such that $\left(x^{-}(t, u, v), y^{-}(t, u, v)\right) \in U_{\delta_{1}}\left(x^{*}, y^{*}\right)$ for any $t \in\left(T(u, v), T(u, v)+t_{1}\right)$.

Suppose that $\left(\eta_{n}\right)$ is a sequence defined as in the proof of Theorem 3.6. Set $A_{k}=$ $\left\{\sigma_{\eta_{k}+1} \in\left(T\left(x_{\eta_{k}}, y_{\eta_{k}}\right), T\left(x_{\eta_{k}}, y_{\eta_{k}}\right)+t_{1}\right)\right\}$. We then have

$$
\begin{aligned}
& \boldsymbol{P}\left(\overline{A_{k}}\right)= \boldsymbol{P}\left\{\sigma_{\eta_{k}+1} \notin\left(T\left(x_{\eta_{k}}, y_{\eta_{k}}\right), T\left(x_{\eta_{k}}, y_{\eta_{k}}\right)+t_{1}\right)\right\} \\
&=\sum_{n=0}^{\infty} \int_{\mathcal{K}_{\varepsilon_{4}}} \boldsymbol{P}\left\{\sigma_{\eta_{k}+1} \notin\left(T\left(x_{\eta_{k}}, y_{\eta_{k}}\right), T\left(x_{\eta_{k}}, y_{\eta_{k}}\right)+t_{1}\right) \mid \eta_{k}=2 n+1, x_{\eta_{k}}=u, y_{\eta_{k}}=v\right\} \\
& \quad \times \boldsymbol{P}\left\{\eta_{k}=2 n+1, x_{\eta_{k}} \in d u, y_{\eta_{k}} \in d v\right\} \\
&=\int_{\mathcal{K}_{\varepsilon_{4}}} \sum_{n=0}^{\infty} \boldsymbol{P}\left\{\sigma_{2 n+2} \notin\left(T(u, v), T(u, v)+t_{1}\right) \mid \eta_{k}=2 n+1, x_{2 n+1}=u, y_{2 n+1}=v\right\} \\
& \quad \times \boldsymbol{P}\left\{\eta_{k}=2 n+1, x_{2 n+1} \in d u, y_{2 n+1} \in d v\right\} .
\end{aligned}
$$

Since $\left(x_{2 n+1}, y_{2 n+1}\right)$ and $\left\{\eta_{k}=2 n+1\right\}$ is $\mathcal{F}_{0}^{2 n+1}$-measurable,

$$
\begin{aligned}
\sum_{n=0}^{\infty} \int_{\mathcal{K}_{\varepsilon_{4}}} \boldsymbol{P}\left\{\sigma_{2 n+2} \notin\left(T(u, v), T(u, v)+t_{1}\right) \mid \eta_{k}=2 n+1, x_{2 n+1}=u, y_{2 n+1}=v\right\} \\
\quad \times \boldsymbol{P}\left\{\eta_{k}=2 n+1, x_{2 n+1} \in d u, y_{2 n+1} \in d v\right\} \\
=\sum_{n=0}^{\infty} \int_{\mathcal{K}_{\varepsilon_{4}}} \boldsymbol{P}\left\{\sigma_{2} \notin\left(T(u, v), T(u, v)+t_{1}\right)\right\} \boldsymbol{P}\left\{\eta_{k}=2 n+1, x_{2 n+1} \in d u, y_{2 n+1} \in d v\right\}
\end{aligned}
$$




$$
\begin{aligned}
=\sum_{n=0}^{\infty} \int_{\mathcal{K}_{\varepsilon_{4}}}\left(1-\boldsymbol{P}\left\{\sigma_{2} \in\left(T(u, v), T(u, v)+t_{1}\right)\right\}\right) \\
\times \boldsymbol{P}\left\{\eta_{k}=2 n+1, x_{2 n+1} \in d u, y_{2 n+1} \in d v\right\} \\
\leq \sum_{n=0}^{\infty} \int_{\mathcal{K}_{\varepsilon_{4}}}\left(1-\boldsymbol{P}\left\{\sigma_{2} \in\left(T_{1}^{*}+2 T, T_{1}^{*}+2 T+t_{1}\right)\right\}\right) \\
\quad \times \boldsymbol{P}\left\{\eta_{k}=2 n+1, x_{2 n+1} \in d u, y_{2 n+1} \in d v\right\} \\
=1-\boldsymbol{P}\left\{\sigma_{2} \in\left(T_{1}^{*}+2 T, T_{1}^{*}+2 T+t_{1}\right)\right\}:=\varphi<1 .
\end{aligned}
$$

Similarly, we can estimate the probability $\boldsymbol{P}\left(\overline{A_{k}} \cap \overline{A_{k+1}}\right)$. For the sake of simplicity we denote $B=\mathcal{K}_{\varepsilon_{4}} \times \mathcal{K}_{\varepsilon_{4}}, C\left(l, n, u, v, u_{1}, v_{1}\right)=\left\{\eta_{k}=2 l+1, \eta_{k+1}=2 n+1, x_{\eta_{k}}=\right.$ $\left.u, y_{\eta_{k}}=v, x_{\eta_{k+1}}=u_{1}, y_{\eta_{k+1}}=v_{1}\right\}$ and $C\left(l, n, d u, d v, d u_{1}, d v_{1}\right)=\left\{\eta_{k}=2 l+1, \eta_{k+1}=\right.$ $\left.2 n+1, x_{\eta_{k}} \in d u, y_{\eta_{k}} \in d v, x_{\eta_{k+1}} \in d u_{1}, y_{\eta_{k+1}} \in d v_{1}\right\}$. We have

$$
\begin{aligned}
& \boldsymbol{P}\left(\overline{A_{k}} \cap \overline{A_{k+1}}\right) \\
& =\boldsymbol{P}\left\{\sigma_{\eta_{k}+1} \notin\left(T\left(x_{\eta_{k}}, y_{\eta_{k}}\right), T\left(x_{\eta_{k}}, y_{\eta_{k}}\right)+t_{1}\right),\right. \\
& \left.\sigma_{\eta_{k+1}+1} \notin\left(T\left(x_{\eta_{k+1}}, y_{\eta_{k+1}}\right), T\left(x_{\eta_{k+1}}, y_{\eta_{k+1}}\right)+t_{1}\right)\right\} \\
& =\sum_{0 \leq l<n<\infty} \int_{B} \boldsymbol{P}\left\{\sigma_{\eta_{k}+1} \notin\left(T\left(x_{\eta_{k}}, y_{\eta_{k}}\right), T\left(x_{\eta_{k}}, y_{\eta_{k}}\right)+t_{1}\right)\right. \text {, } \\
& \sigma_{\eta_{k+1}+1} \notin\left(T\left(x_{\eta_{k+1}}, y_{\eta_{k+1}}\right), T\left(x_{\eta_{k+1}}, y_{\eta_{k+1}}\right)+t_{1}\right) \\
& \left.\mid \eta_{k}=2 l+1, \eta_{k+1}=2 n+1, x_{\eta_{k}}=u, y_{\eta_{k}}=v, x_{\eta_{k+1}}=u_{1}, y_{\eta_{k+1}}=v_{1}\right\} \\
& \times \boldsymbol{P}\left\{\eta_{k}=2 l+1, \eta_{k+1}=2 n+1, x_{\eta_{k}} \in d u, y_{\eta_{k}} \in d v, x_{\eta_{k+1}} \in d u_{1}, y_{\eta_{k+1}} \in d v_{1}\right\} \\
& =\sum_{0 \leq l<n<\infty} \int_{B} \boldsymbol{P}\left\{\sigma_{2 l+2} \notin\left(T(u, v), T(u, v)+t_{1}\right), \sigma_{2 n+2} \notin\left(T\left(u_{1}, v_{1}\right), T\left(u_{1}, v_{1}\right)+t_{1}\right)\right. \\
& \left.\mid C\left(l, n, u, v, u_{1}, v_{1}\right)\right\} \boldsymbol{P}\left\{C\left(l, n, d u, d v, d u_{1}, d v_{1}\right)\right\} \\
& =\sum_{0 \leq l<n<\infty} \int_{B} \boldsymbol{P}\left\{\sigma_{2 n+2} \notin\left(T\left(u_{1}, v_{1}\right), T\left(u_{1}, v_{1}\right)+t_{1}\right)\right\} \boldsymbol{P}\left\{\sigma_{2 l+2} \notin\left(T(u, v), T(u, v)+t_{1}\right)\right. \\
& \text { | } \left.C\left(l, n, u, v, u_{1}, v_{1}\right)\right\} \boldsymbol{P}\left\{C\left(l, n, d u, d v, d u_{1}, d v_{1}\right)\right\} \\
& =\sum_{0 \leq l<n<\infty} \int_{B} \boldsymbol{P}\left\{\sigma_{2} \notin\left(T\left(u_{1}, v_{1}\right), T\left(u_{1}, v_{1}\right)+t_{1}\right)\right\} \boldsymbol{P}\left\{\sigma_{2 l+2} \notin\left(T(u, v), T(u, v)+t_{1}\right)\right. \\
& \text { | } \left.C\left(l, n, u, v, u_{1}, v_{1}\right)\right\} \boldsymbol{P}\left\{C\left(l, n, d u, d v, d u_{1}, d v_{1}\right)\right\} \\
& \leq\left(1-\boldsymbol{P}\left\{\sigma_{2} \in\left(T_{1}^{*}+2 T, T_{1}^{*}+2 T+t_{1}\right)\right\}\right) \sum_{0 \leq l<n<\infty} \int_{B} \boldsymbol{P}\left\{\sigma_{2 l+2} \notin\left(T(u, v), T(u, v)+t_{1}\right)\right. \\
& =\left(1-\boldsymbol{P}\left\{\sigma_{2} \in\left(T_{1}^{*}+2 T, T_{1}^{*}+2 T+t_{1}\right)\right\}\right) \sum_{l=0}^{\infty} \int_{\mathcal{K}_{\varepsilon_{2}}} \boldsymbol{P}\left\{\sigma_{2 l+2} \notin\left(T(u, v), T(u, v)+t_{1}\right)\right. \\
& \left.\mid \eta_{k}=2 l+1, x_{2 l+1}=u, y_{2 l+1}=v\right\} \boldsymbol{P}\left\{\eta_{k}=2 l+1, x_{2 l+1} \in d u, y_{2 l+1} \in d v\right\} \\
& \leq\left(1-\boldsymbol{P}\left\{\sigma_{2} \in\left(T_{1}^{*}+2 T, T_{1}^{*}+2 T+t_{1}\right)\right\}\right)^{2}=\varphi^{2} \text {. }
\end{aligned}
$$


Thus, by induction we obtain

$$
\boldsymbol{P}\left(\bigcap_{i=k}^{n} \overline{A_{i}}\right) \leq\left(1-\boldsymbol{P}\left\{\sigma_{2} \in\left(T_{1}^{*}+2 T, T_{1}^{*}+2 T+t_{1}\right)\right\}\right)^{n-k+1}=\varphi^{n-k+1}
$$

for any $n>k>0$. Hence,

$$
\boldsymbol{P}\left(\bigcap_{k=1}^{\infty} \bigcup_{i=k}^{\infty} A_{i}\right)=\boldsymbol{P}\left\{\omega ; \sigma_{\eta_{n}+1} \in\left(T\left(x_{\eta_{n}}, y_{\eta_{n}}\right), T\left(x_{\eta_{n}}, y_{\eta_{n}}\right)+t_{1}\right) \text { i.o. } n\right\}=1 .
$$

Therefore, there are infinitely many $n$ such that $\sigma_{2 n+2} \in\left(T\left(x_{2 n+1}, y_{2 n+1}\right), T\left(x_{2 n+1}, y_{2 n+1}\right)+\right.$ $\left.t_{1}\right)$, which implies that

$$
\left(x_{2 n+2}, y_{2 n+2}\right)=\left(\left(x^{-}\left(\sigma_{2 n+2}, x_{2 n+1}, y_{2 n+1}\right), y^{-}\left(\sigma_{2 n+2}, x_{2 n+1}, y_{2 n+1}\right)\right) \in U_{\delta_{1}}\left(x^{*}, y^{*}\right) .\right.
$$

The proof for the case $\left(x^{*}, y^{*}\right) \in \gamma^{+}$is similar.

THEOREM 3.8. Any forward orbit of (2.5), starting at a point on $\gamma^{-}$, is a subset of $\omega(x, y)$ with $x>0, y>0$. Similarly, any forward orbit of (2.6), starting at a point on $\gamma^{+}, i$ s a subset of $\omega(x, y)$. (See Figure 1.)

Proof. Suppose that $\gamma^{+}\left(x^{*}, y^{*}\right)$ is the orbit of a solution $\left(x^{+}\left(t, x^{*}, y^{*}\right), y^{+}\left(t, x^{*}, y^{*}\right)\right)$ of (2.5), starting at the point $\left(x^{*}, y^{*}\right) \in \gamma^{-}$. Let $(u, v) \in \gamma^{+}\left(x^{*}, y^{*}\right)$. Then there is $t^{*}>0$ such that $\left(x^{+}\left(t^{*}, x^{*}, y^{*}\right), y^{+}\left(t^{*}, x^{*}, y^{*}\right)\right)=(u, v)$. By the continuous dependence of solutions on the initial data, for any neighborhood $V_{\delta_{1}}$ of $(u, v)$, there are $t_{1}<t^{*}<t_{2}$ and $\delta_{2}>0$ such that if $\left(x_{1}^{*}, y_{1}^{*}\right) \in U_{\delta_{2}}\left(x^{*}, y^{*}\right)$, then $\left(x^{+}\left(t, x_{1}^{*}, y_{1}^{*}\right), y^{+}\left(t, x_{1}^{*}, y_{1}^{*}\right)\right) \in V_{\delta_{1}}(u, v)$ for any $t_{1}<t<t_{2}$. Let

$$
\begin{aligned}
\zeta_{1} & =\inf \left\{2 k ;\left(x_{2 k}, y_{2 k}\right) \in U_{\delta_{2}}\left(x^{*}, y^{*}\right)\right\}, \\
\zeta_{2} & =\inf \left\{2 k>\eta_{1} ;\left(x_{2 k}, y_{2 k}\right) \in U_{\delta_{2}}\left(x^{*}, y^{*}\right)\right\}, \\
& \vdots \\
\zeta_{n} & =\inf \left\{2 k>\eta_{n-1} ;\left(x_{2 k}, y_{2 k}\right) \in U_{\delta_{2}}\left(x^{*}, y^{*}\right)\right\} .
\end{aligned}
$$

By the proof of Lemma 3.7, we see that there are infinitely many even numbers $2 n$ such that $\left(x_{2 n}, y_{2 n}\right) \in U_{\delta_{2}}\left(x^{*}, y^{*}\right)$. Hence, $\zeta_{k}<\infty$ and $\lim _{k \rightarrow \infty} \zeta_{k}=\infty$ a.s. On the other hand, $\left\{\zeta_{k}=n\right\} \in \mathcal{F}_{0}^{n}$ and hence $\left\{\zeta_{k}=n\right\}$ is independent of $\mathcal{F}_{n}^{\infty}$. Therefore,

$$
\begin{aligned}
\boldsymbol{P}\left\{\sigma_{\zeta_{k}+1} \in\left(t_{1}, t_{2}\right)\right\} & =\sum_{n=0}^{\infty} \boldsymbol{P}\left\{\sigma_{\zeta_{k}+1} \in\left(t_{1}, t_{2}\right) \mid \zeta_{k}=2 n\right\} \boldsymbol{P}\left\{\zeta_{k}=2 n\right\} \\
& =\sum_{n=0}^{\infty} \boldsymbol{P}\left\{\sigma_{2 n+1} \in\left(t_{1}, t_{2}\right) \mid \zeta_{k}=2 n\right\} \boldsymbol{P}\left\{\zeta_{k}=2 n\right\} \\
& =\sum_{n=0}^{\infty} \boldsymbol{P}\left\{\sigma_{2 n+1} \in\left(t_{1}, t_{2}\right)\right\} \boldsymbol{P}\left\{\zeta_{k}=2 n\right\} \\
& =\sum_{n=0}^{\infty} \boldsymbol{P}\left\{\sigma_{1} \in\left(t_{1}, t_{2}\right)\right\} \boldsymbol{P}\left\{\zeta_{k}=2 n\right\}=\boldsymbol{P}\left\{\sigma_{1} \in\left(t_{1}, t_{2}\right)\right\}
\end{aligned}
$$


Similarly, we have

$$
\boldsymbol{P}\left\{\sigma_{\zeta_{k}+1} \in\left(t_{1}, t_{2}\right), \sigma_{\zeta_{k+1}+1} \in\left(t_{1}, t_{2}\right)\right\}=\boldsymbol{P}\left\{\sigma_{1} \in\left(t_{1}, t_{2}\right)\right\}^{2},
$$

which implies that

$$
\boldsymbol{P}\left\{\omega ; \sigma_{\zeta_{k}+1} \in\left(t_{1}, t_{2}\right) \text { i.o. } k\right\}=1 .
$$

Since $\left(x_{\zeta_{k}}, y_{\zeta_{k}}\right) \in U_{\delta_{2}}\left(x^{*}, y^{*}\right)$ and $\sigma_{\zeta_{k}+1} \in\left(t_{1}, t_{2}\right)$,

$$
\left(x_{\zeta_{k}+1}, y_{\zeta_{k}+1}\right)=\left(x^{+}\left(\sigma_{\zeta_{k}+1}, x_{\zeta_{k}}, y_{\zeta_{k}}\right), y^{+}\left(\sigma_{\zeta_{k}+1}, x_{\zeta_{k}}, y_{\zeta_{k}}\right)\right) \in V_{\delta_{1}}(u, v)
$$

for infinitely many $k$. This means that $(u, v) \in \omega(x, y)$.

In exactly the same way, we can prove that the orbit $\gamma^{+}\left(x^{*}, y^{*}\right)$ of any solution $\left(x^{*}\left(t, x^{*}\right.\right.$, $\left.\left.y^{*}\right), y^{*}\left(t, x^{*}, y^{*}\right)\right)$ of (2.6) with initial value $\left(x^{*}, y^{*}\right) \in \gamma^{+}$is contained in $\omega(x, y)$.

4. Dynamics of the bistable subsystems. In this section, we keep hypotheses (3.1)(3.3) for $i=+$. Thus, (2.5) has a unique periodic orbit $\gamma^{+}$as in Section 3. For (2.6) we suppose the following.

HYPOTHESIS 4.1.

$$
\begin{aligned}
\inf _{0<t<T} \frac{a(-, t)}{b(-, t)}> & \sup _{0<t<T} \frac{d(-, t)}{e(-, t)}, \sup _{0<t<T} \frac{a(-, t)}{c(-, t)}<\inf _{0<t<T} \frac{d(-, t)}{f(-, t)}, \\
& \sup _{0<t<T} \frac{b(-, t)}{e(-, t)}<\inf _{0<t<T} \frac{c(-, t)}{f(-, t)} .
\end{aligned}
$$

By virtue of condition (4.1), we can choose constants $k_{1}^{-}$and $k_{2}^{-}$satisfying

$$
\frac{a(-, t)}{b(-, t)}>k_{1}^{-}>\frac{d(-, s)}{e(-, s)}, \quad \frac{d(-, t)}{f(-, t)}>k_{2}^{-}>\frac{a(-, s)}{c(-, s)}
$$

for any $s, t \in \boldsymbol{R}$. Further, there is a unique periodic orbit, say $\gamma_{U}^{-}$, of the marginal equation

$$
\dot{u}^{-}=u^{-}\left(a(-, t)-b(-, t) u^{-}\right),
$$

and a unique periodic orbit, say $\gamma_{V}^{-}$, of the marginal equation

$$
\dot{v}^{-}=v^{-}\left(d(-, t)-f(-, t) v^{-}\right) .
$$

Moreover, it is easy to see that $\gamma_{U}^{-} \subset\left[k_{1}^{-}, \infty\right)$ and it attracts any solution $u^{-}(t)$ with $u^{-}(0)>0$. Similarly, $\gamma_{V}^{-} \subset\left[k_{2}^{-}, \infty\right)$ and it attracts any solution $v^{-}(t)$ with $v^{-}(0)>0$ (see Lemma 2.1). We now come to the investigation of some properties of the solution of (2.6).

LEMMA 4.2. Under conditions (4.1) and (4.2), there exists a function $\varphi:\left[0, a^{*}\right) \rightarrow$ $\boldsymbol{R}^{+}$such that if $y<\varphi(x)$, then $\lim _{t \rightarrow \infty} y^{-}(t, x, y)=0$ and $\lim _{t \rightarrow \infty}\left(x^{-}(t, x, y)-u^{-}(t, x)\right)=$ 0 . Conversely, if $y>\varphi(x)$, then $\lim _{t \rightarrow \infty} x^{-}(t, x, y)=0$ and $\lim _{t \rightarrow \infty}\left(y^{-}(t, x, y)-v^{-}(t, y)\right)=$ 0 . Thus, there is a 'neutral' curve, say $\ell$, such that if $\left(x_{0}, y_{0}\right) \in \ell$, then the solution $\left(x^{-}\left(t, x_{0}, y_{0}\right)\right.$, $\left.y^{-}\left(t, x_{0}, y_{0}\right)\right)$ is bounded above and below by positive constants. Furthermore, there is a unique periodic orbit contained in $\ell$, say $\gamma^{-}$, which is visited by any solution starting at a 
point on $\ell$, i.e., for any $\delta>0,\left(x^{*}, y^{*}\right) \in \gamma^{-}$, there is a $t>0$ such that $\left(x^{-}\left(t, x_{0}, y_{0}\right)\right.$, $\left.y^{-}\left(t, x_{0}, y_{0}\right)\right) \in U_{\delta}\left(x^{*}, y^{*}\right)$ for any $\left(x_{0}, y_{0}\right) \in \ell$.

Proof. See [6, Proposition 2.10]. We prove the last assertion on the existence of a periodic orbit. If $(x, y) \in \ell$, then $\left(x^{-}(t, x, y), y^{-}(t, x, y)\right) \in \ell$ for any $t>0$. We consider the map $(x, y) \mapsto\left(x^{-}(T, x, y), y^{-}(T, x, y)\right)$ from $\ell$ into $\ell$. It is easy to prove that this map has a fixed point by Brouwer's fixed point theorem. Therefore, there is a periodic orbit, say $\gamma^{-}$. This orbit is unique because there is only one solution bounded above and below by positive constants. Finally, the visitation of any point on $\gamma^{-}$follows from the uniqueness of $\gamma^{-}$and the periodicity of coefficients.

Note that we have $x(t) \uparrow$ and $y(t) \uparrow($ resp. $x(t) \downarrow$ and $y(t) \downarrow)$ whenever $x(t)$ and $y(t)$ of the solution of (2.4) are both small (resp. whenever at least one of $x(t)$ and $y(t)$ is large). Thus, for sufficiently small $\varepsilon$ chosen in (3.7), we can find $t_{0} \geq 0$ such that $x(t)<M / m, y(t)<$ $M / m$ and either $x(t) \geq \varepsilon$ or $y(t) \geq \varepsilon$ for any $t \geq t_{0}$. Therefore, without loss of generality, we may suppose that $x(t)<M / m, y(t)<M / m$ and either $x(t) \geq \varepsilon$ or $y(t) \geq \varepsilon$ for any $t \geq 0$.

Put

$$
\mathcal{A}=\{(x, y) ; y<\varphi(x), x>0\}, \quad \mathcal{B}=\{(x, y) ; y>\varphi(x), x>0\}
$$

with the convention $\varphi(x)=\infty$ if $x \geq a^{*}$.

LEMMA 4.3. For any compact set $K \subset \mathcal{A}$ (resp. $K \subset \mathcal{B}$ ) and any $\delta_{3}$-neighborhood $U_{\delta_{3}}\left(\gamma_{U}^{-} \times\{0\}\right)$ of $\gamma_{U}^{-} \times\{0\}$ (resp. $\delta_{3}$-neighborhood $V_{\delta_{3}}\left(\{0\} \times \gamma_{V}^{-}\right)$of $\left.\{0\} \times \gamma_{V}^{-}\right)$, there is a $T_{3}^{*}=T_{3}^{*}\left(\delta_{3}, K\right)>0$ such that $\left(x^{-}(t, x, y), y^{-}(t, x, y)\right) \in U_{\delta_{3}}\left(\gamma_{U}^{-} \times\{0\}\right)$ for any $t>T_{3}^{*}$ (resp. $\left(x^{-}(t, x, y), y^{-}(t, x, y)\right) \in V_{\delta_{3}}\left(\{0\} \times \gamma_{V}^{-}\right)$for any $\left.t>T_{3}^{*}\right)$ and for any $(x, y) \in K$.

Proof. Let $K \subset \mathcal{A}$ and $(x, y) \in K$. Then by Lemma 4.2, there is a $T=T(x, y)$ such that $\left(x^{-}(t, x, y), y^{-}(t, x, y)\right) \in U_{\delta_{3}}$ for all $t \geq T(x, y)$. By the continuous dependence of solutions on the initial data, there exists an open neighborhood $U_{x, y}$ of $(x, y)$ such that $\left(x^{-}(t, u, v), y^{-}(t, u, v)\right) \in U_{\delta_{3}}$ for all $t \geq T(x, y)$ and $(u, v) \in U_{x, y}$. The family $\left(U_{x, y}\right)_{(x, y) \in K}$ is an open covering of $K$. Since $K$ is compact, there are $U_{x_{i}, y_{i}}, i=1, \ldots, n$, such that $K \subset \bigcup_{i=1}^{n} U_{x_{i}, y_{i}}$. Putting $T_{3}^{*}=\max _{1 \leq i \leq n} T\left(x_{i}, y_{i}\right)$, we have the result. The case $K \subset \mathcal{B}$ is treated in a similar way.

LEMMA 4.4. Let $\left(x^{-}(t, x, y), y^{-}(t, x, y)\right)$ be the solution of (2.6). For any $\varepsilon_{1}>0$ with $[\varepsilon, \infty) \times\left[0, \varepsilon_{1}\right] \subset \mathcal{A}$, we can find $\varepsilon_{3}>0$ such that if $\left(x^{-}\left(t_{1}\right), y^{-}\left(t_{1}\right)\right) \in[\varepsilon, \infty) \times\left[0, \varepsilon_{3}\right]$, then $x^{-}(t) \geq \varepsilon$ for any $t \geq t_{1}$ and $\sup _{t>t_{1}} y^{-}(t) \leq \varepsilon_{1}$. A similar result holds for the case of $\left(x^{-}\left(t_{1}\right), y^{-}\left(t_{1}\right)\right) \in\left[0, \varepsilon_{1}\right] \times[\varepsilon, \infty)$.

PRoOF. In a manner similar to that in the proof of Lemma 4.3, we can find $T_{3}^{*}=$ $T_{3}^{*}\left(\varepsilon_{1} / 2,\left\{[\varepsilon, M / m] \times\left[0, \varepsilon_{1}\right]\right\}\right)$ such that $\left(x^{-}(t, x, y), y^{-}(t, x, y)\right) \in U_{\varepsilon_{1} / 2}\left(\gamma_{U}^{-} \times\{0\}\right)$ for any $t>T_{3}^{*}$. Hence, by choosing $\varepsilon_{3}=\varepsilon_{1} e^{-M T_{3}^{*}}$, we have $\dot{y}^{-}=y^{-}\left(d(-, t)-e(-, t) x^{-}-\right.$ $\left.f(-, t) y^{-}\right)<y^{-} M$, which implies that

$$
y^{-}(t)<\max \left\{y^{-}\left(t_{1}\right) e^{M T_{3}^{*}}, \varepsilon_{1} / 2\right\} \leq \max \left\{\varepsilon_{3} e^{M T_{3}^{*}}, \varepsilon_{1} / 2\right\}=\varepsilon_{1} .
$$


LEMMA 4.5. There is $S_{1}>0$ such that if $\varepsilon \leq y(t)$ (resp. $\left.\varepsilon \leq x(t)\right)$, then $\inf _{0<s<S_{1}} y(t+$ $s) \geq \varepsilon / 2\left(\right.$ resp. $\left.\inf _{0<s<S_{1}} x(t+s) \geq \varepsilon / 2\right)$ for any $t \geq 0$.

Proof. On the set $(0, M / m] \times(0, M / m], \dot{y} / y$ is bounded below by a constant, say $\gamma$, and hence $y(t+s) \geq y(t) e^{\gamma s}$. We can choose $0<S_{1}<|\ln 2 / \gamma|$.

LEMMA 4.6. The image of the periodic solution $u^{*}(t)$ of (2.7) is the smallest interval, denoted by $\Gamma_{x}$, containing $\gamma_{U}^{+}$and $\gamma_{U}^{-}$. Similarly, the image of the periodic solution $v^{*}(t)$ of (2.8) is the smallest interval, denoted by $\Gamma_{y}$, containing $\gamma_{V}^{+}$and $\gamma_{V}^{-}$. Here, $\gamma_{U}^{+}, \gamma_{V}^{+}$are two periodic orbits of $\dot{u}^{+}=u^{+}\left(a(+, t)-b(+, t) u^{+}\right)$and $\dot{v}^{+}=v^{+}\left(d(+, t)-f(+, t) v^{+}\right)$, respectively.

Proof. The function $t \mapsto u^{*}(t)$ is continuous and it is easy to show that the $\omega$-limit set of $u^{*}(t)$ contains $\gamma_{U}^{+}, \gamma_{U}^{-}$with probability one. Therefore, its image is a connected set in $\boldsymbol{R}^{+}$. Thus it must be an interval. Hence, by Lemma 2.1, this image is the smallest interval containing $\gamma_{U}^{+}, \gamma_{U}^{-}$. The argument is similar for the solution $v^{*}(t)$.

THEOREM 4.7. Suppose that conditions (2.10) and (2.11) hold. Let $\omega(x, y)$ be the $\omega$-limit set of the solution $(x(t, x, y), y(t, x, y))$ of (2.4) with $x>0, y>0$. Then, $\gamma^{+} \subset$ $\omega(x, y)$, where $\gamma^{+}$is a unique periodic solution of $(2.5)$.

PROOF. The proof is somewhat similar to that of Theorem 3.6. For the convenience of the proof, we suppose $\xi_{0}=+$. Let

$$
\varepsilon_{1}=\min \left\{\varepsilon, \frac{\lambda}{2 M}, \frac{\gamma}{2 M}\right\}, \quad \varepsilon_{4}=\min \left\{\varepsilon_{2}, \varepsilon_{3}\right\}
$$

where $\varepsilon_{2}$ as mentioned in Corollary 3.4 is chosen so that $\varepsilon_{2}<\varepsilon_{1}$ and $\varepsilon_{3}$ is given by Lemma 4.4. We construct a sequence

$$
\begin{aligned}
& \eta_{1}=\inf \left\{2 k+1 ;\left(x_{2 k+1} \geq \varepsilon \wedge y_{2 k+1} \geq \varepsilon_{4}\right) \text { or }\left(x_{2 k+1} \geq \varepsilon_{4} \wedge y_{2 k+1} \geq \varepsilon\right)\right\}, \\
& \eta_{2}=\inf \left\{2 k+1>\eta_{1} ;\left(x_{2 k+1} \geq \varepsilon \wedge y_{2 k+1} \geq \varepsilon_{4}\right) \text { or }\left(x_{2 k+1} \geq \varepsilon_{4} \wedge y_{2 k+1} \geq \varepsilon\right)\right\}, \\
& \quad \vdots \\
& \eta_{n}=\inf \left\{2 k+1>\eta_{n-1} ;\left(x_{2 k+1} \geq \varepsilon \wedge y_{2 k+1} \geq \varepsilon_{4}\right) \text { or }\left(x_{2 k+1} \geq \varepsilon_{4} \wedge y_{2 k+1} \geq \varepsilon\right)\right\} .
\end{aligned}
$$

Put $A_{n}=\left\{\omega ; \sigma_{\eta_{n}+1}<s\right.$ and $\left.\sigma_{\eta_{n}+2}>t\right\}$. By the same trick as used in the proof of Theorem 3.6 we can show that

$$
\boldsymbol{P}\left(\bigcap_{k=1}^{\infty} \bigcup_{i=k}^{\infty} A_{i}\right)=\boldsymbol{P}\left\{\omega ; \sigma_{\eta_{n}+1}<s \text { and } \sigma_{\eta_{n}+2}>t \text { i.o. } n\right\}=1 .
$$

Suppose that $\delta_{1}>0$ is arbitrary and $U_{\delta_{2}}\left(\gamma^{+}\right)$is a neighborhood of $\gamma^{+}$as in Lemma 3.5. We choose $S_{1}$ as in Lemma 4.5 and $T_{1}^{*}=T_{1}^{*}\left(\delta_{2}, \varepsilon_{4} / 2\right)$ as in Lemma 3.2. By choosing $s=S_{1}$ and $t=T_{1}^{*}+2 T$ in (4.4), we see that there are infinitely many $n$ such that either $\left(x_{2 n+1} \geq \varepsilon \wedge y_{2 n+1} \geq \varepsilon_{4}\right)$ or $\left(x_{2 n+1} \geq \varepsilon_{4} \wedge y_{2 n+1} \geq \varepsilon\right)$ with $\sigma_{2 n+2}<S_{1}$ and $\sigma_{2 n+3}>T_{1}^{*}+2 T$. Using Lemma 4.5 , we obtain $\left(x_{2 n+2}, y_{2 n+2}\right) \in \mathcal{K}_{\varepsilon_{4} / 2}$, which implies that $\left(x\left(\tau_{2 n+2}+T_{3}^{*}, x, y\right), y\left(\tau_{2 n+2}+T_{3}^{*}, x, y\right)\right)=\left(x^{+}\left(T_{3}^{*}, x_{2 n+2}, y_{2 n+2}\right), y^{+}\left(T_{3}^{*}, x_{2 n+2}, y_{2 n+2}\right)\right)$ $\in U_{\delta_{2}}\left(\gamma^{+}\right)$by Lemma 3.2. Thus, from Lemma 3.5, for any $\left(x^{*}, y^{*}\right) \in \gamma^{+}$, the trajectory 
of $(x(t, x, y), y(t, x, y))$ passes through the neighborhood $U_{\delta_{1}}\left(x^{*}, y^{*}\right)$ on the interval $\left(\tau_{2 n+2}, \tau_{2 n+3}\right)$. This means that $\gamma^{+} \subset \omega(x, y)$.

LEMMA 4.8. If $\left(x^{*}, y^{*}\right) \in \gamma^{+}$, then for any $\delta_{1}>0$ we have

$$
\boldsymbol{P}\left\{\left(x_{n}, y_{n}\right) \in U_{\delta_{1}}\left(x^{*}, y^{*}\right) \text { i.o. } n\right\}=1 .
$$

PROOF. The proof is quite similar to that of Lemma 3.7, so we omit it.

THEOREM 4.9. Suppose that conditions (2.10) and (2.11) hold. Let $\omega(x, y)$ be the $\omega$-limit set of the solution $(x(t, x, y), y(t, x, y))$ with $x>0, y>0$.

(a) Every orbit $\gamma^{-}\left(x^{*}, y^{*}\right)$ of the solution of (2.6) starting at any point of $\gamma^{+}$is a subset of $\omega(x, y)$.

(b) If $\gamma^{+} \cap \mathcal{A} \neq \emptyset$, then $\Gamma_{x} \times\{0\} \subset \omega(x, y)$

(c) If $\gamma^{+} \cap \mathcal{B} \neq \emptyset$, then $\{0\} \times \Gamma_{y} \subset \omega(x, y)$.

(d) If $C:=\gamma^{+} \cap \ell \neq \emptyset$, then the smallest connected part of $\ell$ containing $\gamma^{-}$(a unique periodic orbit contained in $\ell$, see Lemma 4.2) and $C$ is contained in $\omega(x, y)$.

Proof. (a) We obtain the assertion by replacing Lemma 3.7 with Lemma 4.8 in the proof of Theorem 3.8.

(b) Suppose that $\left(x^{*}, y^{*}\right) \in \gamma^{+} \cap \mathcal{A}$. From (a) we see that $\gamma^{-}\left(x^{*}, y^{*}\right) \subset \omega(x, y)$. Since $\left(x^{*}, y^{*}\right) \in \mathcal{A}$, by Lemmas 2.1 and 4.2 we see that $\gamma_{U}^{-} \times\{0\} \subset \gamma^{-}\left(x^{*}, y^{*}\right)$. Thus, $\gamma_{U}^{-} \times\{0\} \subset \omega(x, y)$.

Let $x_{1}^{*} \in \gamma_{U}^{+}$and $\delta_{1}>0$ be arbitrary. It is easy to see that $\gamma_{U}^{+} \subset\left[\varepsilon, k_{1}^{+}\right]$and there is $T_{4}^{*}>0$ such that $\gamma_{U}^{+} \subset u_{\left[t_{1}, t_{1}+T_{4}^{*}\right]}^{+}\left(\cdot, u_{0}\right)$ for any $M / m>u_{0}>\varepsilon$, where $u_{\left[t_{1}, t_{1}+T_{4}\right]}^{+}\left(\cdot, u_{0}\right)=$ $\left\{u^{+}\left(t, u_{0}\right) ; t_{1} \leq t \leq t_{1}+T_{4}\right\}$. Hence, by the continuous dependence of solutions on the initial data, we can find $\delta_{2}>0$ such that if $(u, v) \in U_{\delta_{2}}\left(\gamma_{U}^{-} \times\{0\}\right)$, we have

$$
\left\{\left(x^{+}(t, u, v), y^{+}(t, u, v) ; t \in\left[t_{1}, t_{1}+T_{4}^{*}\right]\right\} \cap U_{\delta_{1}}\left(x_{1}^{*}, 0\right) \neq \emptyset .\right.
$$

Given $\xi_{0}=+$, we set

$$
\begin{aligned}
& \zeta_{1}=\inf \left\{2 k ;\left(x_{2 k}, y_{2 k}\right) \in U_{\delta_{2}}\left(\gamma_{U}^{-} \times\{0\}\right)\right\}, \\
& \zeta_{2}=\inf \left\{2 k>\zeta_{1} ;\left(x_{2 k}, y_{2 k}\right) \in U_{\delta_{2}}\left(\gamma_{U}^{-} \times\{0\}\right)\right\}, \\
& \quad \vdots \\
& \zeta_{n}=\inf \left\{2 k>\zeta_{n-1} ;\left(x_{2 k}, y_{2 k}\right) \in U_{\delta_{2}}\left(\gamma_{U}^{-} \times\{0\}\right)\right\} .
\end{aligned}
$$

Since $\gamma_{U}^{-} \times\{0\} \subset \omega(x, y), \zeta_{k}<\infty$ for any $k$ and $\lim _{k \rightarrow \infty} \zeta_{k}=\infty$ a.s. Moreover, $\left\{\zeta_{k}=n\right\}$ is independent of $\mathcal{F}_{n}^{\infty}$. Therefore, by the same argument as above

$$
\boldsymbol{P}\left\{\omega ; \sigma_{\zeta_{k}+1} \geq T_{4}^{*} \text { i.o. } k\right\}=1 .
$$

Thus, there are infinitely many $n$ such that $\left(x_{2 n}, y_{2 n}\right) \in U_{\delta_{2}}\left(\gamma_{U}^{-} \times\{0\}\right)$ and $\sigma_{2 n+1} \geq T_{4}^{*}$. Hence, from (4.6) we have $\left\{(x(t, x, y), y(t, x, y)) ; t \in\left[\tau_{2 n}, \tau_{2 n}+T_{4}^{*}\right]\right\} \cap U_{\delta_{1}}\left(x_{1}^{*}, 0\right) \neq \emptyset$, which means that $\left(x_{1}^{*}, 0\right) \in \omega(x, y)$.

(c) The proof is similar. 
(d) First, we prove that $\gamma^{-} \subset \omega(x, y)$. Let $\delta>0$ be arbitrary, $\left(x^{*}, y^{*}\right) \in \gamma^{-}$and $\left(x_{1}^{*}, y_{1}^{*}\right) \in C$. Since any point of $\gamma^{-}$is visited, there is $t^{*}>0$ such that $\left(x^{-}\left(t^{*}, x_{1}^{*}, y_{1}^{*}\right)\right.$, $\left.y^{-}\left(t^{*}, x_{1}^{*}, y_{1}^{*}\right)\right) \in U_{\delta}\left(x^{*}, y^{*}\right)$. By the continuous dependence of solutions on the initial data, there are $t_{1}<t^{*}<t_{2}$ and $\delta_{1}>0$ such that $\left(x^{-}(t, u, v), y^{-}(t, u, v)\right) \in U_{\delta}\left(x^{*}, y^{*}\right)$ for any $t \in\left(t_{1}, t_{2}\right)$ and $(u, v) \in U_{\delta_{1}}\left(x_{1}^{*}, y_{1}^{*}\right)$. On the other hand, in the same way as above and by virtue of Lemma 4.8, it follows that there are infinitely many odd numbers $n$ such that $\left(x_{n}, y_{n}\right) \in U_{\delta_{1}}\left(x_{1}^{*}, y_{1}^{*}\right)$ and $\sigma_{n+1} \in\left(t_{1}, t_{2}\right)$ with probability 1 . Hence, $\left(x_{n+1}, y_{n+1}\right)=$ $\left(x^{-}\left(\sigma_{n+1}, x_{n}, y_{n}\right), y^{-}\left(\sigma_{n+1}, x_{n}, y_{n}\right)\right) \in U_{\delta}\left(x^{*}, y^{*}\right)$ with probability 1, i.e., $\left(x^{*}, y^{*}\right) \in \omega(x, y)$.

It is obvious that $C \cap \ell \subset \omega(x, y)$. Then, by noting that $(x(t, x, y), y(t, x, y))$ is continuous, we see that the smallest part containing $C$ and $\gamma^{-}$is included in $\omega(x, y)$ with probability 1 .

We illustrate the above-mentioned model by the following numerical examples.

EXAMPLE I. Figure 2 illustrates an example of the system satisfying $\gamma^{+} \cap \mathcal{A}=\emptyset$ and $\gamma^{+} \cap \mathcal{B} \neq \varnothing$ (see Theorems 4.7 and 4.9(a) and (c)).

EXAMPLE II. Figure 3 illustrates an example of the system satisfying $\gamma^{+} \cap \mathcal{A} \neq \varnothing$ and $\gamma^{+} \cap \mathcal{B} \neq \emptyset$ (see Theorems 4.7 and 4.9(a)-(d)).

5. Discussion. To conclude this paper, we consider an ecology system of two competing species. Suppose that the evolution of each species depends on the quantity of rainfall for every period. If the rainfall is sufficient, their competition potential is equal and they develop periodically. Whenever the rainfall is small, the second species becomes very weak and its amount gets smaller with the increase of time although the influence of the other environmental elements is still seasonal (periodical). However, in the case when the rainfall is in a stationary regime, the quantity of each species oscillates between the good situation and the bad situation. Neither of the species disappears.

There are some questions here. In the proof of Theorem 3.6 we suppose that conditions (2.10) and (2.11) hold. However, if we use the Liapunov function $V^{+}=m y^{\gamma}$ and $V^{-}=n y^{\gamma}$ with $m, n, \gamma$ chosen appropriately, we can prove that if $0<y(0)<\varepsilon$ and $0<x(0)<r_{1}$ with a positive probability, then $(x(t), y(t))$ has to get out from the domain $0<y \leq \varepsilon$, i.e., there exist $t^{*}>0$ such that $y\left(t^{*}\right)>\varepsilon$, with a positive probability. However, we need that $(x(t), y(t))$ gets out from the domain $0<y \leq \varepsilon$ with probability 1 . Thus, we use the assumptions (2.10) and (2.11) perhaps only for technical reasons. As suggested by Figure 1, we conjecture that Theorem 3.6 is still true without these assumptions. Moreover, whether there is or is not a Markov periodic solution with period $T$ that attracts all the solutions of (2.4) with initial data in $\boldsymbol{R}_{+} \times \boldsymbol{R}_{+}$under conditions (3.1)-(3.3) is an open question.

Also, Figure 1 suggests that the system composed of two stable subsystems is permanent under conditions (2.10) and (2.11). Note that Theorem 2.3 implies only that the system is average permanent. We intend to study this problem in the future. 
(a)

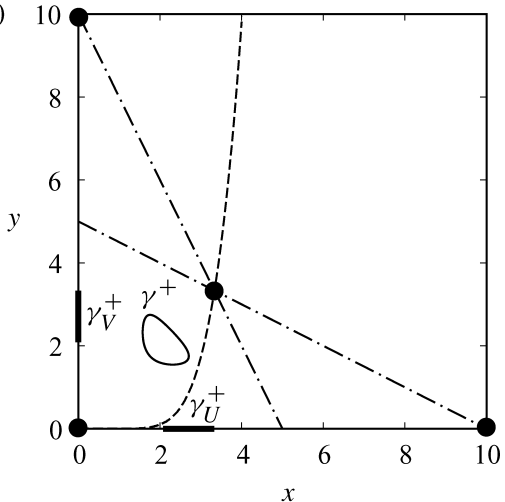

(b)

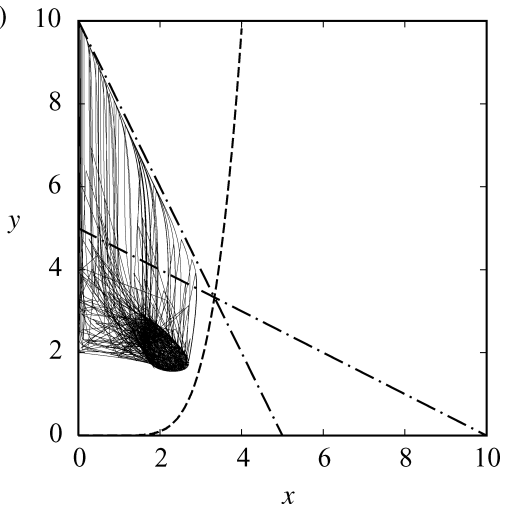

Figure 2. The $x-y$ phase planes. (a) The periodic solutions $\left(\gamma^{+}, \gamma_{U}^{+}\right.$and $\left.\gamma_{V}^{+}\right)$of system (2.5) with the following parameters are plotted: $a(+, t)=10, b(+, t)=$ $\sin (t)+4, c(+, t)=1, d(+, t)=10$ $e(+, t)=1, f(+, t)=\sin (t+\pi / 2)+$ 4. For system (2.6) with the following parameters, null-clines (dot-dashed lines), equilibrium points (solid dots) and a neutral curve $\ell$ (a broken line) are shown: $a(-, t)=2, b(-, t)=0.2$, $c(-, t)=0.4, d(-, t)=2, e(-, t)=$ $0.4, f(-, t)=0.2$. (b) The solution of system (2.4) switching between the above systems (2.5) and (2.6) with the initial condition $(x(0), y(0))=(4,0.2)$ is plotted for $t \in[800,1000]$. The transition intensities are $\alpha=1$ and $\beta=1$.

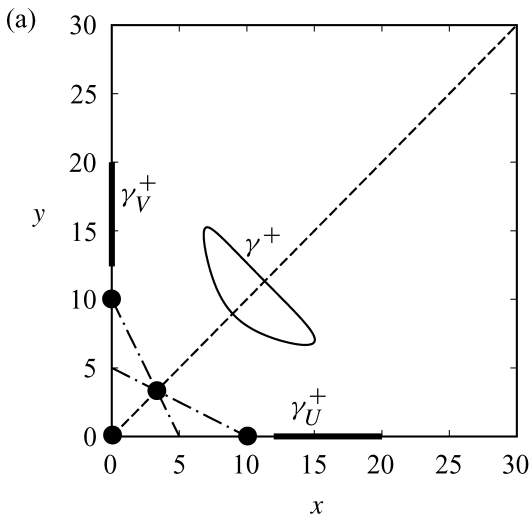

(b)

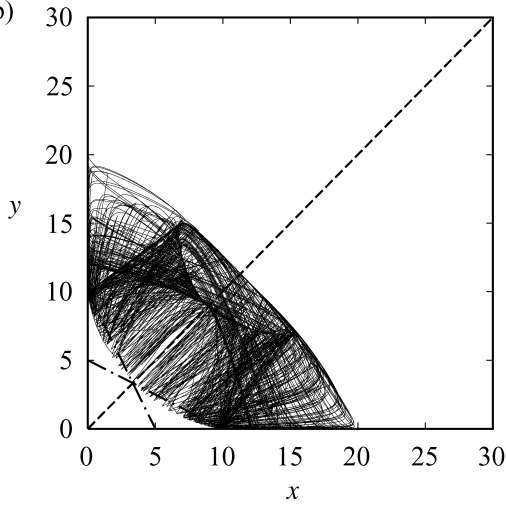

FIGURE 3. The $x-y$ phase planes. (a) The periodic solutions $\left(\gamma^{+}, \gamma_{U}^{+}\right.$and $\left.\gamma_{V}^{+}\right)$of system (2.5) with the following parameters are plotted: $a(+, t)=30, b(+, t)=$ $0.5(\sin (t)+4), c(+, t)=1, d(+, t)=$ $30, e(+, t)=1, f(+, t)=0.5(\sin (t+$ $\pi / 2)+4)$. For system (2.6) with the following parameters, null-clines (dotdashed lines), equilibrium points (solid dots) and a neutral curve $\ell$ (a broken line) are shown: $a(-, t)=6, b(-, t)=$ $0.6, c(-, t)=1.2, d(-, t)=6$, $e(-, t)=1.2, f(-, t)=0.6$. (b) The solution of system (2.4) switching between the above systems (2.5) and (2.6) with the initial condition $(x(0), y(0))=$ $(4,0.2)$ is plotted for $t \in[1300,2000]$. The transition intensities are $\alpha=1$ and $\beta=1$. 
ACKNOWLEDGMENTS. The first author expresses his gratitude for the support of JSPS under grant AP 1012571. He is also grateful to the Department of Systems Engineering, Shizuoka University for its generous hospitality during his stay. The last author's work is partly supported by the Ministry of Education, Science and Culture in Japan under the Grandin-Aid for Scientific Research (A) 13304006.

\section{REFERENCES}

[ 1 ] L. ARnold, Random dynamical systems, Springer Monogr. Math., Springer, Berlin, 1998.

[2] S. AhmAD, Extinction of species in nonautonomous Lotka-Volterra systems, Proc. Amer. Math. Soc. 127 (1999), 2905-2910.

[ 3 ] S. Ahmad, On the nonautonomous Volterra-Lotka competition equations, Proc. Amer. Math. Soc. 117 (1993), 199-204.

[ 4 ] P. L. CHESSON AND R. R. WARNER, Environmental variability promotes coexistence in lottery competitive systems, Amer. Natur. 117 (1981), 923-943.

[ 5 ] N. H. Du, On the existence of bounded solutions for Lotka-Volterra equations, Acta Math. Vietnam 25 (2000), $145-159$.

[ 6 ] N. H. Du, R. Kon, K. SATO AND Y. TAKEUCHI, Dynamical behavior of Lotka-Volterra competition systems: nonautonomous bistable case and the effect of telegraph noise, J. Comput. Appl. Math. 170 (2004), 399-422.

[ 7 ] M. FArKas, Periodic motions, Appl. Math. Sci. 104, Springer, New York, 1994.

[ 8 ] I. I. Gihman And A. V. Skorohod, The theory of Stochastic processes III, Springer-Verlag, Berlin-New York, 1979

[ 9 ] I. Hanski, P. Turchin, E. Korpimäki and H. Henttonen, Population oscillations of boreal rodents: regulation by mustelid predators leads to chaos, Nature 364 (1994), 232-235.

[10] J. HofBAuER AND K. Sigmund, Evolutionary games and population dynamics, Cambridge University Press, Cambridge, 1998.

[11] M. LoREAU, Coexistence of temporally segregated competitors in a cyclic environment, Theoret. Population Biol. 36 (1989), 181-201.

[12] R. S. Lipshter And Shyriaev, Statistics of Stochastic processes, Nauka, Moscow (in Russian), 1974

[13] T. NAmbA AND S. TAKAHASHI, Competitive coexistence in a seasonally fluctuating environment II. Multiple stable states and invasion success, Theoret. Population Biol. 44 (1993) 374-402.

[14] Y. TAKeUCHI, Global dynamical properties of Lotka-Volterra systems, World Scientific Publishing Co., Inc., River Edge, N.J., 1996.

FACULTY OF MATHEMATICS

MECHANICS AND INFORMATICS

HANOI NATIONAL UNIVERSITY

334 NGUYEN TRAI

THANH XUAN, HANOI

VIETNAM
FACUlTy OF MATHEMATICS

KYUSHU UNIVERSITY

FUKUOKA 812-858

JAPAN

E-mail address: kon-r@math.kyushu-u.ac.jp 
DEPARTMENT OF SYSTEMS ENGINEERING

SHIZUOKA UNIVERSITY

HAMAMATSU 432-8561

JAPAN

E-mail address: sato@sys.eng.shizuoka.ac.jp
DEPARTMENT OF SYSTEMS ENGINEERING

SHIZUOKA UNIVERSITY

HAMAMATSU 432-8561

JAPAN

E-mail address: takeuchi@sys.eng.shizuoka.ac.jp 